\title{
Data-driven design of targeted gene panels for estimating immunotherapy biomarkers
}

\author{
Jacob R. Bradley and Timothy I. Cannings \\ School of Mathematics, University of Edinburgh
}

\begin{abstract}
We introduce a novel data-driven framework for the design of targeted gene panels for estimating exome-wide biomarkers in cancer immunotherapy. Our first goal is to develop a generative model for the profile of mutation across the exome, which allows for gene- and variant type-dependent mutation rates. Based on this model, we then propose a new procedure for estimating biomarkers such as Tumour Mutation Burden and Tumour Indel Burden. Our approach allows the practitioner to select a targeted gene panel of a prespecified size, and then construct an estimator that only depends on the selected genes. Alternatively, the practitioner may apply our method to make predictions based on an existing gene panel, or to augment a gene panel to a given size. We demonstrate the excellent performance of our proposal using data from three Non-Small Cell Lung Cancer studies, as well as data from six other cancer types.

Keywords: cancer, gene panel design, targeted sequencing, tumour indel burden, tumour mutation burden.
\end{abstract}

\section{Introduction}

It has been understood for a long time that cancer, a disease occurring in many distinct tissues of the body and giving rise to a wide range of presentations, is initiated and driven by the accumulation of mutations in a subset of a person's cells (Boveri, 2008). Since the discovery of Immune Checkpoint Blockade (ICB) ${ }^{1}$ (Ishida et al., 1992; Leach et al., 1996), there has been an explosion of interest in cancer therapies targeting immune response and ICB therapy is now widely used in clinical practice (Robert, 2020). ICB therapy works by targeting natural mechanisms (or checkpoints) that disengage the immune system, for example the proteins Cytotoxic T Lymphocyte Associated protein 4 (CTLA-4) and Programmed Death Ligand 1 (PD-L1) (Buchbinder and Desai, 2016). Inhibition of these checkpoints can promote a more aggressive anti-tumour immune response (Pardoll, 2012), and in some patients this leads to long-term remission (Borghaei et al., 2021). However, ICB therapy is not always effective (Nowicki et al., 2018) and may have adverse side-effects, so determining which patients will benefit in advance of treatment is vital.

Exome-wide prognostic biomarkers for immunotherapy are now well-established - in particular, Tumour Mutation Burden (TMB) is used to predict response to immunotherapy

\footnotetext{
${ }^{1}$ For their work on ICB, James Allison and Tasuku Honjo received the 2018 Nobel Prize for Physiology/Medicine (Ledford et al., 2018).
} 
(Zhu et al., 2019; Cao et al., 2019). TMB is defined as the total number of non-synonymous mutations occurring throughout the tumour exome, and can be thought of as a proxy for how easily a tumour cell can be recognised as foreign by immune cells (Chan et al., 2019). However, the cost of measuring TMB using Whole Exome Sequencing (WES) (Sboner et al., 2011) currently prohibits its widespread use as standard-of-care. Sequencing costs, both financial and in terms of the time taken for results to be returned, are especially problematic in situations where high-depth sequencing is required, such as when utilising blood-based circulating tumour DNA (ctDNA) from liquid biopsy samples (Gandara et al., 2018). The same issues are encountered when measuring more recently proposed biomarkers such as Tumour Indel Burden (TIB) (Wu et al., 2019a; Turajlic et al., 2017), which counts the number of frameshift insertion and deletion mutations. There is, therefore, demand for costeffective approaches to estimate these biomarkers (Fancello et al., 2019; Golkaram et al., 2020).

In this paper we propose a novel, data-driven method for biomarker estimation, based on a generative model of how mutations arise in the tumour exome. More precisely, we model mutation counts as independent Poisson variables, where the mean number of mutations depends on the gene of origin and variant type, as well as the Background Mutation Rate (BMR) of the tumour. Due to the ultrahigh-dimensional nature of sequencing data and the fact that in many genes mutations arise purely according to the BMR, we use a regularisation penalty when estimating the parameters of the model. In addition, this identifies a subset of genes that are mutated above or below the background rate. Our model facilitates the construction of a new estimator of TMB, based on a weighted linear combination of the number of mutations in each gene. The vector of weights is chosen to be sparse (i.e. have many entries equal to zero), so that our estimator of TMB may be calculated using only the mutation counts in a subset of genes. In particular, this allows for accurate estimation of TMB from a targeted gene panel, where the panel size (and therefore the cost) may be determined by the user.

We demonstrate the excellent practical performance of our framework using a Non-Small Cell Lung Cancer (NSCLC) dataset (Campbell et al., 2016), and include a comparison with existing state-of-the-art approaches for estimating TMB. We further validate these results by testing the performance on data from two more NSCLC studies (Hellmann et al., 2018b; Rizvi et al., 2015). Moreover, since our model allows variant type-dependent mutation rates, it can be adapted easily to predict other biomarkers, such as TIB. Our method may also be used in combination with an existing targeted gene panel. In particular, we can estimate a biomarker directly from the panel, or first augment the panel and then construct an estimator. Finally, in order to further investigate the utility of our proposal across a range of mutation profiles, we use it to select targeted gene panels and estimate TMB in six other cancer types.

Due to its emergence as a biomarker for immunotherapy in recent years, a variety of groups have considered methods for estimating TMB. A simple and common way to estimate TMB is via the proportion of mutated codons in a targeted region. Budczies et al. (2019) investigate how the accuracy of predictions made in this way are affected by the size of the targeted region, where mutations are assumed to occur at uniform rate throughout the genome. More recently Yao et al. (2020) modelled mutations as following a negative binomial distribution while allowing for gene-dependent rates, which are inferred by comparing nonsynonymous and synonymous mutation counts. In contrast, our method does not require 
data including synonymous mutations. Where they are included, we do not assume that synonymous mutations occur at a uniform rate throughout the genome, giving us the flexibility to account for location-specific effects on synonymous mutation rate such as chromatin configuration (Makova and Hardison, 2015) and transcription-dependent repair mechanisms (Fong et al., 2013). Linear regression models have been used for both panel selection (Lyu et al., 2018) and for biomarker prediction (Guo et al., 2020). A review of some of the issues arising when dealing with targeted panel-based predictions of TMB biomarkers is given by Wu et al. (2019b). Finally, we are unaware of any methods for estimating TIB from targeted gene panels.

The remainder of the paper is as follows. In Section 2, we introduce our NSCLC data sources, and provide a detailed description of our methodological proposal. The full demonstration of our method using the NSCLC dataset is given in Section 3. Section 4 provides several further analyses to investigate the robustness of our proposal in other cancer types and we conclude in Section 5. We also provide an R package ICBioMark (Bradley and Cannings, 2021) which implements the methodology and reproduces the experimental results in the paper.

\section{Methodology}

\subsection{Data and terminology}

Our methodology can be applied to any annotated mutation dataset obtained by WES. To demonstrate our proposal we make use of the NSCLC dataset produced by Campbell et al. (2016), which contains data from 1144 patient-derived tumours. For each sample in this dataset we have the genomic locations and variant types of all mutations identified. At the time of the study, the patients had a variety of prognoses and smoking histories, were aged between 39 and 90, 41\% were female and 59\% were male; see Figure 1. In Figure 2A we see that mutations counts are distributed over a very wide range, as is the case in many cancer types (Chalmers et al., 2017). For simplicity, we only consider seven nonsynonymous variant types: missense mutations (which are the most abundant), nonsense mutations, frameshift insertions/deletions, splice site mutations, in-frame insertions/deletions, nonstop mutations and translation start site mutations. We present the frequencies of these mutation types in Figure 2B. Frameshift insertion/deletion (also known as indel) mutations are of particular interest when predicting TIB, but contribute only a small proportion $(<4 \%)$ of nonsynonymous mutations.

It is useful at this point to introduce the notation used throughout the paper. The set $G$ denotes the collection of genes that make up the exome. For a gene $g \in G$, let $\ell_{g}$ be the length of $g$ in nucleotide bases, defined by the maximum coding sequence ${ }^{2}$. A gene panel is a subset $P \subseteq G$, and we write $\ell_{P}:=\sum_{g \in P} \ell_{g}$ for its total length. We let $S$ denote the set of variant types in our data (e.g. in the dataset mentioned above, $S$ contains the seven possible non-synonymous variants). Now, for $i=0,1, \ldots, n$, let $M_{i g s}$ denote the count of mutations in gene $g \in G$ of type $s \in S$ in the $i$ th sample. Here the index $i=0$ is used

\footnotetext{
${ }^{2}$ The maximum coding sequence is defined as the collection of codons that may be translated for some version of a gene, even if all the codons comprising the maximum coding sequence are never simultaneously translated. Gene coding lengths are extracted from the Ensembl database (Yates et al., 2020).
} 

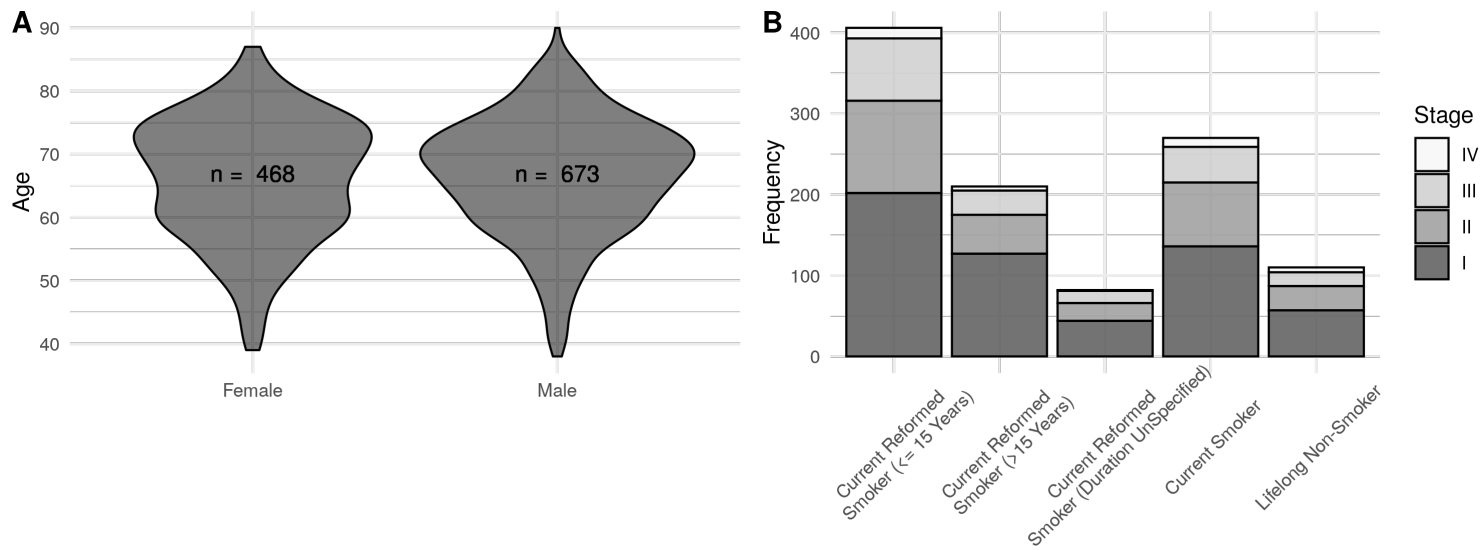

Figure 1: Demographic data for the clinical cohort in Campbell et al. (2016). A: Violin plots of age for patients, stratified by sex. B: Stacked bar chart of patients' smoking histories, shaded according to cancer stage diagnosis.
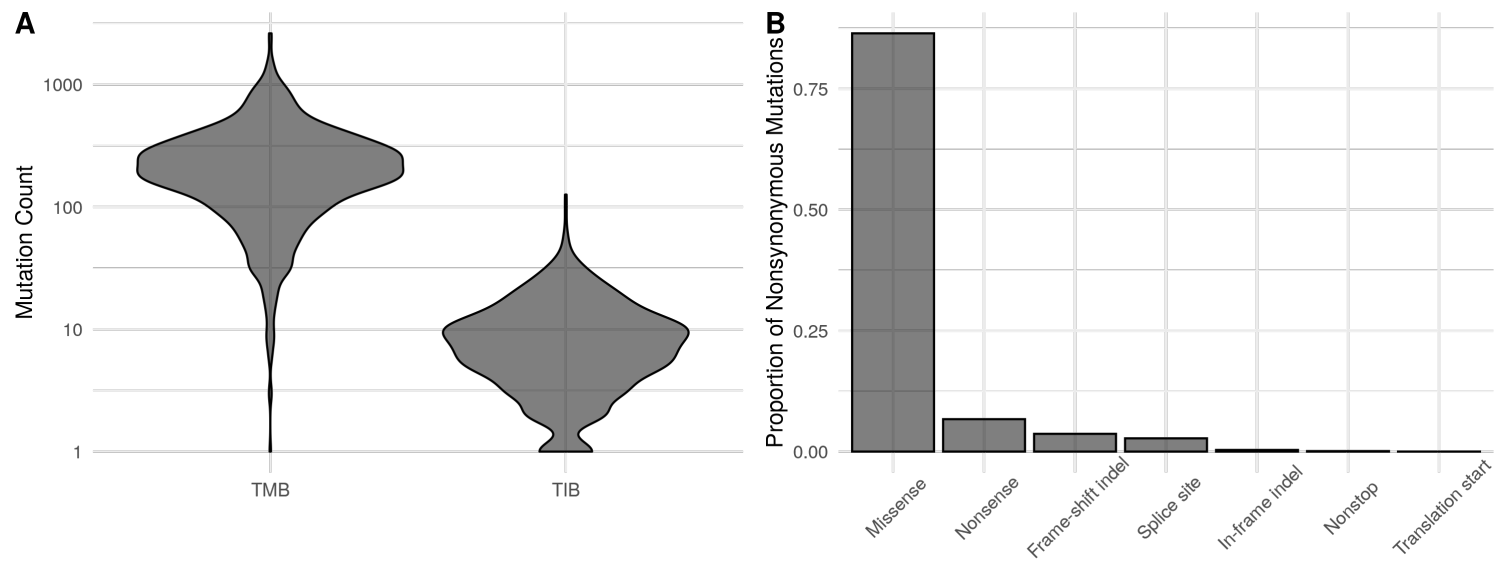

Figure 2: Dataset-wide distribution of mutations. A: Violin plot of the distribution of TMB and TIB across training samples. B: The relative frequency of different nonsynonymous mutation types. 
to refer to an unseen test sample for which we would like to make a prediction, while the indices $i=1, \ldots, n$ enumerate the samples in our training data set. In order to define the exome-wide biomarker of particular interest, we specify a subset of mutation types $\bar{S} \subseteq S$, and let

$$
T_{i \bar{S}}:=\sum_{g \in G} \sum_{s \in \bar{S}} M_{i g s},
$$

for $i=0, \ldots, n$. For example, including all non-synonymous mutation types in $\bar{S}$ specifies $T_{i \bar{S}}$ as the TMB of sample $i$, whereas letting $\bar{S}$ contain only indel mutations gives TIB.

Our main goal is to predict $T_{0 \bar{S}}$ based on $\left\{M_{0 g s}: g \in P, s \in S\right\}$, where the panel $P \subseteq G$ has length $\ell_{P}$ satisfying some upper bound. When it is clear from context that we are referring to the test sample and a specific choice of biomarker (i.e. $\bar{S}$ is fixed), we will simply write $T$ in place of $T_{0 \bar{S}}$.

\subsection{Generative model}

We now describe the main statistical model that underpins our methodology. In order to account for selective pressures and other factors within the tumour, we allow the rate at which mutations occur to depend on the gene and type of mutation. Our model also includes a sample-dependent parameter to account for the differing levels of mutagenic exposure of tumours, which may occur due to exogenous (e.g. UV light, cigarette smoke) or endogenous (e.g. inflammatory, free radical) factors.

We model the mutation counts $M_{i g s}$ as independent Poisson random variables with mutation rates $\phi_{i g s}>0$. More precisely, for $i=0,1, \ldots, n, g \in G$ and $s \in S$, we have

$$
M_{i g s} \sim \operatorname{Poisson}\left(\phi_{i g s}\right)
$$

where $M_{i g s}$ and $M_{i^{\prime} g^{\prime} s^{\prime}}$ are independent for $(i, g, s) \neq\left(i^{\prime}, g^{\prime}, s^{\prime}\right)$. Further, to model the dependence of the mutation rate on the sample, gene and mutation type, we use a log link function and let

$$
\log \left(\phi_{i g s}\right)=\mu_{i}+\log \left(\ell_{g}\right)+\lambda_{g}+\nu_{s}+\eta_{g s},
$$

for $\mu_{i}, \lambda_{g}, \nu_{s}, \eta_{g s} \in \mathbb{R}$, where for identifiability we set $\eta_{g s_{1}}=0$, for some $s_{1} \in S$ and all $g \in G$.

The terms in our model can be interpreted as follows. First, the parameter $\mu_{i}$ corresponds to the BMR of the $i$ th sample. The offset $\log \left(\ell_{g}\right)$ accounts for a mutation rate that is proportional to the length of a gene, so that a non-zero value of $\lambda_{g}$ corresponds to increased or decreased mutation rate relative to the BMR. The parameters $\nu_{s}$ and $\eta_{g s}$ account for differences in frequency between mutation types for each gene.

The model in (2) and (3) (discounting the unseen test sample $i=0$ ) has $n+|S|+|G||S|$ free parameters and we have $n|G||S|$ independent observations in the training data set. In principle we could attempt to fit our model directly using maximum likelihood estimation. However, we wish to exploit the fact that most genes do not play an active role in the development of a tumour, and will be mutated approximately according to the BMR. This corresponds to the parameters $\lambda_{g}$ and $\eta_{g s}$ being zero for many $g \in G$. We therefore include an $\ell_{1}$-penalisation term applied to the parameters $\lambda_{g}$ and $\eta_{g s}$ when fitting our model. We do not penalise the parameters $\nu_{s}$ or $\mu_{i}$ since we expect that different mutation types occur at different rates, and that the BMR is different in each sample. 
Writing $\mu:=\left(\mu_{1}, \ldots, \mu_{n}\right), \lambda:=\left(\lambda_{g}: g \in G\right), \nu:=\left(\nu_{s}: s \in S\right)$ and $\eta:=\left(\eta_{g s}: g \in G, s \in\right.$ $S)$, and given training observations $M_{i g s}=m_{i g s}$, we let

$$
\mathcal{L}(\mu, \lambda, \nu, \eta)=\sum_{i=1}^{n} \sum_{g \in G} \sum_{s \in S}\left(\phi_{\text {igs }}-m_{\text {igs }} \log \phi_{\text {igs }}\right)
$$

be the negative log-likelihood of the model specified by (2) and (3). We then define

$$
(\hat{\mu}, \hat{\lambda}, \hat{\nu}, \hat{\eta})=\underset{\mu, \lambda, \nu, \eta}{\arg \min }\left\{\mathcal{L}(\mu, \lambda, \nu, \eta)+\kappa_{1}\left(\sum_{g \in G}\left|\lambda_{g}\right|+\sum_{g \in G} \sum_{s \in S}\left|\eta_{g s}\right|\right)\right\},
$$

where $\kappa_{1} \geq 0$ is a tuning parameter that controls the number of non-zero components in $\hat{\lambda}$ and $\hat{\eta}$, which we choose using cross-validation (see Section 2.5 for more detail).

\subsection{Proposed estimator}

We now attend to our main goal of estimating a given exome-wide biomarker for the unseen test sample. Fix $\bar{S} \subseteq S$ and recall that we write $T=T_{0 \bar{S}}$. We wish to construct an estimator of $T$ that only depends on the mutation counts in a gene panel $P \subset G$, subject to a constraint on $\ell_{P}$. To that end, we consider estimators of the form ${ }^{3}$

$$
T(w):=\sum_{g \in G} \sum_{s \in S} w_{g s} M_{0 g s}
$$

for $w \in \mathbb{R}^{|G| \times|S|}$. In the remainder of this subsection we explain how the weights $w$ are chosen to minimise the expected squared error of $T(w)$ based on the generative model in Section 2.2.

Of course, setting $w_{g s}=1$ for $g \in G$ and $s \in \bar{S}$ (and $w_{g s}=0$ otherwise) will give $T(w)=T$. However, our aim is to make predictions based on a concise gene panel. If, for a given $g \in G$, we have $w_{g s}=0$ for all $s \in S$, then $T(w)$ does not depend on the mutations in $g$ and therefore the gene does not need to be included in the panel. In order to produce a suitable gene panel (i.e. with many $w_{g s}=0$ ), we penalise non-zero components of $w$ when minimising the expected squared error. We define our final estimator via a refitting procedure, which improves the predictive performance by reducing the bias, and is also helpful when applying our procedure to panels with predetermined genes.

To construct our estimator, note that under our model in (2) we have $\mathbb{E} M_{0 g s}=\operatorname{Var}\left(M_{0 g s}\right)=$ $\phi_{0 g s}$, and it follows that the expected squared error of $T(w)$ is

$$
\begin{aligned}
\mathbb{E}\left[\{T(w)-T\}^{2}\right]= & \operatorname{Var}(T(w))+\operatorname{Var}(T)-2 \operatorname{Cov}(T(w), T)+[\mathbb{E}\{T(w)-T\}]^{2} \\
= & \sum_{g \in G} \sum_{s \in \bar{S}}\left(1-w_{g s}\right)^{2} \phi_{0 g s}+\sum_{g \in G} \sum_{s \in S \backslash \bar{S}} w_{g s}^{2} \phi_{0 g s} \\
& \quad+\left(\sum_{g \in G} \sum_{s \in S} w_{g s} \phi_{0 g s}-\sum_{g \in G} \sum_{s \in \bar{S}} \phi_{0 g s}\right)^{2} .
\end{aligned}
$$

\footnotetext{
${ }^{3}$ Note that our estimator may use the the full set $S$ of variant types, rather than just those in $\bar{S}$. In other words, our estimator may utilise information from every mutation type, not just those that directly constitute the biomarker of interest. This is important when estimating mutation types in $\bar{S}$ that are relatively scarce (e.g. for TIB).
} 
This depends on the unknown parameters $\mu_{0}, \lambda_{g}, \nu_{s}$ and $\eta_{g s}$, the latter three of which are replaced by their estimates given in (4). It is also helpful to then rescale (5) as follows: write $\hat{\phi}_{0 g s}=\ell_{g} \exp \left(\hat{\lambda}_{g}+\hat{\nu}_{s}+\hat{\eta}_{g s}\right)$, and define

$$
p_{g s}:=\frac{\hat{\phi}_{0 g s}}{\sum_{g^{\prime} \in G} \sum_{s^{\prime} \in \bar{S}} \hat{\phi}_{0 g^{\prime} s^{\prime}}}=\frac{\ell_{g} \exp \left(\hat{\lambda}_{g}+\hat{\nu}_{s}+\hat{\eta}_{g s}\right)}{\sum_{g^{\prime} \in G} \sum_{s^{\prime} \in \bar{S}} \ell_{g^{\prime}} \exp \left(\hat{\lambda}_{g^{\prime}}+\hat{\nu}_{s^{\prime}}+\hat{\eta}_{g^{\prime} s^{\prime}}\right)} .
$$

Then let

$$
f(w):=\sum_{g \in G} \sum_{s \in \bar{S}} p_{g s}\left(1-w_{g s}\right)^{2}+\sum_{g \in G} \sum_{s \in S \backslash \bar{S}} p_{g s} w_{g s}^{2}+K\left(\mu_{0}\right)\left(1-\sum_{g \in G} \sum_{s \in S} p_{g s} w_{g s}\right)^{2},
$$

where $K\left(\mu_{0}\right)=\exp \left(\mu_{0}\right) \sum_{g \in G} \sum_{s \in \bar{S}} \ell_{g} \exp \left(\hat{\lambda}_{g}+\hat{\nu}_{s}+\hat{\eta}_{g s}\right)$. Since $f$ is a rescaled version of the error in (5) (with the true parameters $\lambda, \nu, \eta$ replaced by the estimates $\hat{\lambda}, \hat{\nu}, \hat{\eta}$ ), we will choose $w$ to minimise $f(w)$.

Note that $f$ only depends on $\mu_{0}$ via the $K\left(\mu_{0}\right)$ term, which can be interpreted as a penalty factor controlling the bias of our estimator. For example, we may insist that the squared bias term $\left(1-\sum_{g \in G} \sum_{s \in S} p_{g s} w_{g s}\right)^{2}$ is zero by setting $K\left(\mu_{0}\right)=\infty$. In practice, we propose to choose the penalty $K$ based on the training data; see Section 2.5 .

At this point $f(w)$ is minimised by choosing $w$ to be such that $w_{g s}=1$ for all $g \in G, s \in \bar{S}$, and $w_{g s}=0$ otherwise. As mentioned above, in order to form a concise panel while optimising predictive performance, we impose a constraint on the cost of sequencing the genes used in the estimation. More precisely, for a given $w$, an appropriate cost is

$$
\|w\|_{G, 0}:=\sum_{g \in G} \ell_{g} \mathbb{1}\left\{w_{g s} \neq 0 \text { for some } s \in S\right\} .
$$

This choice acknowledges that the cost of a panel is roughly proportional to the length of the region of genomic space sequenced, and that once a gene has been sequenced for one mutation type there is no need to sequence again for other mutation types.

Now, given a cost restriction $L$, our goal is to minimise $f(w)$ such that $\|w\|_{G, 0} \leq L$. In practice this problem is non-convex and so computationally infeasible. As is common in high-dimensional optimisation problems, we consider a convex relaxation as follows: let $\|w\|_{G, 1}:=\sum_{g \in G} \ell_{g}\left\|w_{g}\right\|_{2}$, where $w_{g}=\left(w_{g s}: s \in S\right) \in \mathbb{R}^{|S|}$, for $g \in G$, and $\|\cdot\|_{2}$ is the Euclidean norm. Define

$$
\hat{w}^{\text {first-fit }} \in \underset{w}{\arg \min }\left\{f(w)+\kappa_{2}\|w\|_{G, 1}\right\},
$$

where $\kappa_{2} \geq 0$ is chosen to determine the size of the panel selected.

The final form of our estimator is obtained by a refitting procedure. First, for $P \subseteq G$, let

$$
W_{P}:=\left\{w \in \mathbb{R}^{|G| \times|S|}: w_{g}=(0, \ldots, 0) \text { for } g \in G \backslash P\right\} .
$$

Let $\hat{P}:=\left\{g \in G:\left\|\hat{w}_{g}^{\text {first-fit }}\right\|_{2}>0\right\}$ be the panel selected by the first-fit estimator in (6), and define

$$
\hat{w}^{\text {refit }} \in \underset{w \in W_{\hat{P}}}{\arg \min }\{f(w)\} .
$$

We then estimate $T$ using $\hat{T}:=T\left(\hat{w}^{\text {refit }}\right)$, which only depends on mutations in genes contained in the selected panel $\hat{P}$. The performance of our estimator is investigated in Section 3, for comparison we also include the performance of the first-fit estimator $T\left(\hat{w}^{\mathrm{first}}\right.$-fit $)$. 


\subsection{Panel augmentation}

In practice, when designing gene panels a variety of factors contribute to the choice of genes included. For example, a gene may be included due to its relevance to immune response or its known association with a particular cancer type. If this is the case, measurements for these genes will be made regardless of their utility for predicting exome-wide biomarkers. When implementing our methodology, therefore, there is no additional cost to incorporate observations from these genes into our prediction if they will be helpful. Conversely researchers may wish to exclude genes from a panel, or at least from actively contributing to the estimation of a biomarker, for instance due to technical difficulties in sequencing a particular gene.

We can accommodate these restrictions by altering the structure of our regularisation penalty in (6). Suppose we are given (disjoint sets of genes) $P_{0}, Q_{0} \subseteq G$ to be included and excluded from our panel, respectively. In this case, we replace $\hat{w}^{\text {first-fit }}$ in (6) with

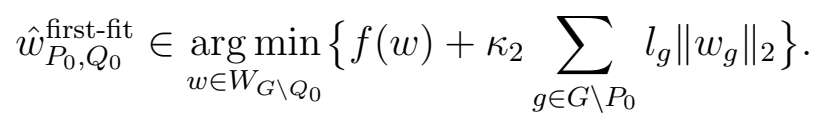

Excluding the elements of $P_{0}$ from the penalty term means that $\hat{w}_{P_{0}, Q_{0}}^{\text {first-fit }} \neq 0$ for the genes in $P_{0}$, while restricting our optimisation to $W_{G \backslash Q_{0}}$ excludes the genes in $Q_{0}$ by definition. This has the effect of augmenting the predetermined panel $P_{0}$ with additional genes selected to improve predictive performance. We then perform refitting as described above. We demonstrate this procedure by augmenting the TST-170 gene panel in Section 3.5.

\subsection{Practical considerations}

In this section, we discuss some practical aspects of our proposal. Our first consideration concerns the choice of the tuning parameter $\kappa_{1}$ in (4). As is common for the Least Absolute Shrinkage and Selection Operator (LASSO) estimator in generalised linear regression (see, for example, Michoel (2016) and Friedman et al. (2021)), we will use 10-fold cross-validation. To highlight one important aspect of our cross-validation procedure, recall that we consider the observations $M_{i g s}$ as independent across the sample index $i \in\{1, \ldots, n\}$, the gene $g \in G$ and the mutation type $s \in S$. Our approach therefore involves splitting the entire set $\{(i, g, s): i=1, \ldots, n, g \in G, s \in S\}$ of size $n|G||S|$ (as opposed to the sample set $\{1, \ldots, n\}$ ) into 10 folds uniformly at random. We then apply the estimation method in (4) to each of the 10 folds separately on a grid of values (on the log scale) of $\kappa_{1}$, and select the value that results in the smallest average deviance across the folds. The model is then refitted using all the data for this value of $\kappa_{1}$.

The estimated coefficients in (6) depend on the choice of $K\left(\mu_{0}\right)$ and $\kappa_{2}$. As mentioned above, we could set $K\left(\mu_{0}\right)=\infty$ to give an unbiased estimator, however in practice we found that a finite choice of $K\left(\mu_{0}\right)$ leads to improved predictive performance. Our recommendation is to use $K\left(\mu_{0}\right)=K\left(\max _{i=1, \ldots, n}\left\{\hat{\mu}_{i}\right\}\right)$, where $\hat{\mu}_{i}=\log \left(T_{i} / \sum_{g, s} \ell_{g} \exp \left(\hat{\lambda}_{g}+\hat{\nu}_{s}+\hat{\eta}_{g s}\right)\right)$ is a pseudo-MLE (in the sense of Gong and Samaniego (1981)) for $\mu_{i}$, so that the penalisation is broadly in proportion with the largest values of $\mu_{i}$ in the training dataset. The tuning parameter $\kappa_{2}$ controls the size of the gene panel selected in (6): given a panel length $L$, we set $\kappa_{2}(L)=\max \left\{\kappa_{2}: \ell_{\hat{P}} \leq L\right\}$ in order to produce a suitable panel.

We now comment briefly on some computational aspects of our method. The generative model fit in (4) can be solved via coordinate descent (see, for example, Friedman et al., 2010), 
which has a computational complexity of $O\left(N|G|^{2}|S|^{2}\right)$ per iteration. We fit the model 10 times, one for each fold in our cross-validation procedure. This is the most computationally demanding part of our proposal - in our experiments below, it takes approximately an hour to solve on a standard laptop - but it only needs to be carried out once for a given dataset. The convex optimisation problem in (6) can be solved by any method designed for the group LASSO; see, for example, Yang and Zou (2015). In our experiments in Section 3, we use the gglasso R package (Yang et al., 2020), which takes around 10 minutes to reproduce the plot in Figure 6. Note also that the solutions to (6) and (8) are unique; see, for example, Roth and Fischer (2008, Theorem 1). The last step of our proposal, namely making predictions for new test observations based on a selected panel, carries negligible computational cost.

Finally we describe a heuristic procedure for producing prediction intervals around our point estimates. In particular, for a given confidence level $\alpha \in(0,1)$, we aim to find an interval $\left[\hat{T}_{\mathrm{L}}, \hat{T}_{\mathrm{U}}\right]$ such that $\mathbb{P}\left(\hat{T}_{\mathrm{L}} \leq T \leq \hat{T}_{\mathrm{U}}\right) \geq 1-\alpha$. To that end, let $t_{\alpha}:=\mathbb{E}\left\{(\hat{T}-T)^{2}\right\} / \alpha$, then by Markov's inequality we have that $\mathbb{P}\left(|\hat{T}-T|^{2} \geq t_{\alpha}\right) \leq \alpha$. It follows that $\left[\hat{T}-t_{\alpha}^{1 / 2}, \hat{T}+\right.$ $\left.t_{\alpha}^{1 / 2}\right]$ is a $(1-\alpha)$-prediction interval for $T$. Of course, the mean squared error $\mathbb{E}\left\{(\hat{T}-T)^{2}\right\}$ defined in (5) depends on the parameters $\lambda, \eta, \nu$ and $\mu_{0}$, which are unknown. Our approach is to utilise the estimates $\hat{\lambda}, \hat{\eta}, \hat{\nu}$ (see (4)) and replace $\mu_{0}$ with $\log \left(\hat{T} / \sum_{g, s} \ell_{g} \exp \left(\hat{\lambda}_{g}+\hat{\nu}_{s}+\hat{\eta}_{g s}\right)\right)$. While this is not an exact $(1-\alpha)$-prediction interval for $T$, we will see in our experimental results in Sections 3.2 and 3.4 that in practice this approach provides intervals with valid empirical coverage.

\section{Demonstration using an NSCLC dataset}

In this section we demonstrate the practical performance of our proposal using the dataset from Campbell et al. (2016), which we introduced in Section 2.1. Our main focus is the prediction of TMB, and we show that our approach outperforms the state-of-the-art approaches. We also analyse the suitability of our generative model, consider the task of predicting the recently proposed biomarker TIB, and include a panel augmentation case study with the TST-170 gene panel.

Since we are only looking to produce estimators for TMB and TIB, we group mutations into two categories - indel mutations and all other non-synonymous mutations - so that $|S|=2$. This simplifies the presentation of our results and reduces the computational cost of fitting the generative model. In order to assess the performance of each of the methods in this section, we randomly split the dataset into training, validation and test sets, which contain $n_{\text {train }}=n=800, n_{\text {val }}=171$ and $n_{\text {test }}=173$ samples, respectively. Mutations are observed in $|G|=17358$ genes. Our training set comprises samples with an average TMB of 252 and TIB of 9.25 .

\subsection{Generative model fit and validation}

The first step in our analysis is to fit the model proposed in Section 2.2 using only the training dataset. In particular, we obtain estimates of the model parameters using equation (4), where the tuning parameter $\kappa_{1}$ is determined using 10-fold cross-validation as described in Section 2.5. The results are presented in Figure 3. The best choice of $\kappa_{1}$ produces estimates of $\lambda$ and $\eta$ with $44.4 \%$ and $77.8 \%$ sparsity respectively, i.e. that proportion of their components 
are estimated to be exactly zero. We plot $\hat{\lambda}$ and $\hat{\eta}$ for this value of $\kappa_{1}$ in Figures 4 and 5 . Genes with $\hat{\lambda}_{g}=0$ are interpreted to be mutating according to the background mutation rate, and genes with $\hat{\eta}_{\text {g,indel }}=0$ are interpreted as having no specific selection pressure for or against indel mutations. In Figures 4 and 5 we highlight genes with large (in absolute value) parameter estimates, some of which have known biological relevance in oncology; see Section 5 for further discussion. Finally, note that the average $\mu_{i}$ among current smokers is 5.40 (with standard deviation 0.76), amongst reformed smokers is 5.26 (0.84), and among lifelong non-smokers is 4.04 (1.12). This suggest that smokers may have higher BMRs, which is as we expect.

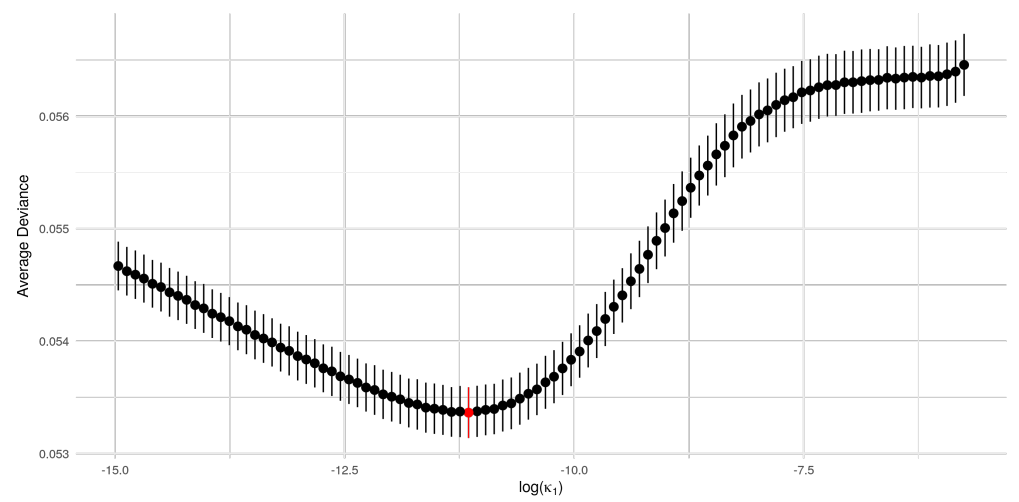

Figure 3: The average deviance (with one standard deviation) across the 10 folds in our crossvalidation procedure plotted against $\log \left(\kappa_{1}\right)$. The minimum average deviance is highlighted red.
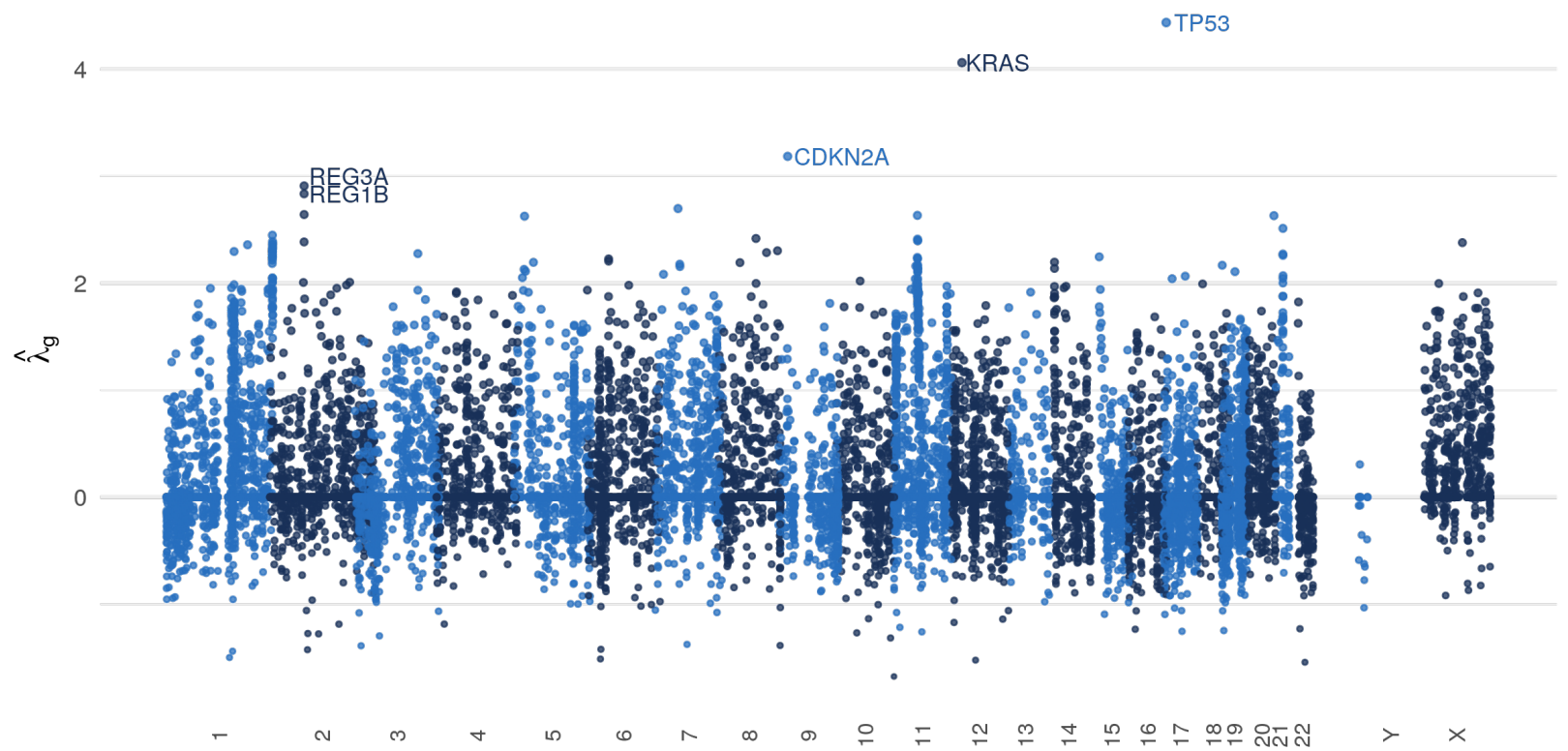

Figure 4: Manhattan plot of fitted parameters $\hat{\lambda}_{g}$ and their associated genes' chromosomal locations. The genes with the five largest positive parameter estimates are labelled.

We now validate our model in (3) by comparing with the following alternatives: 


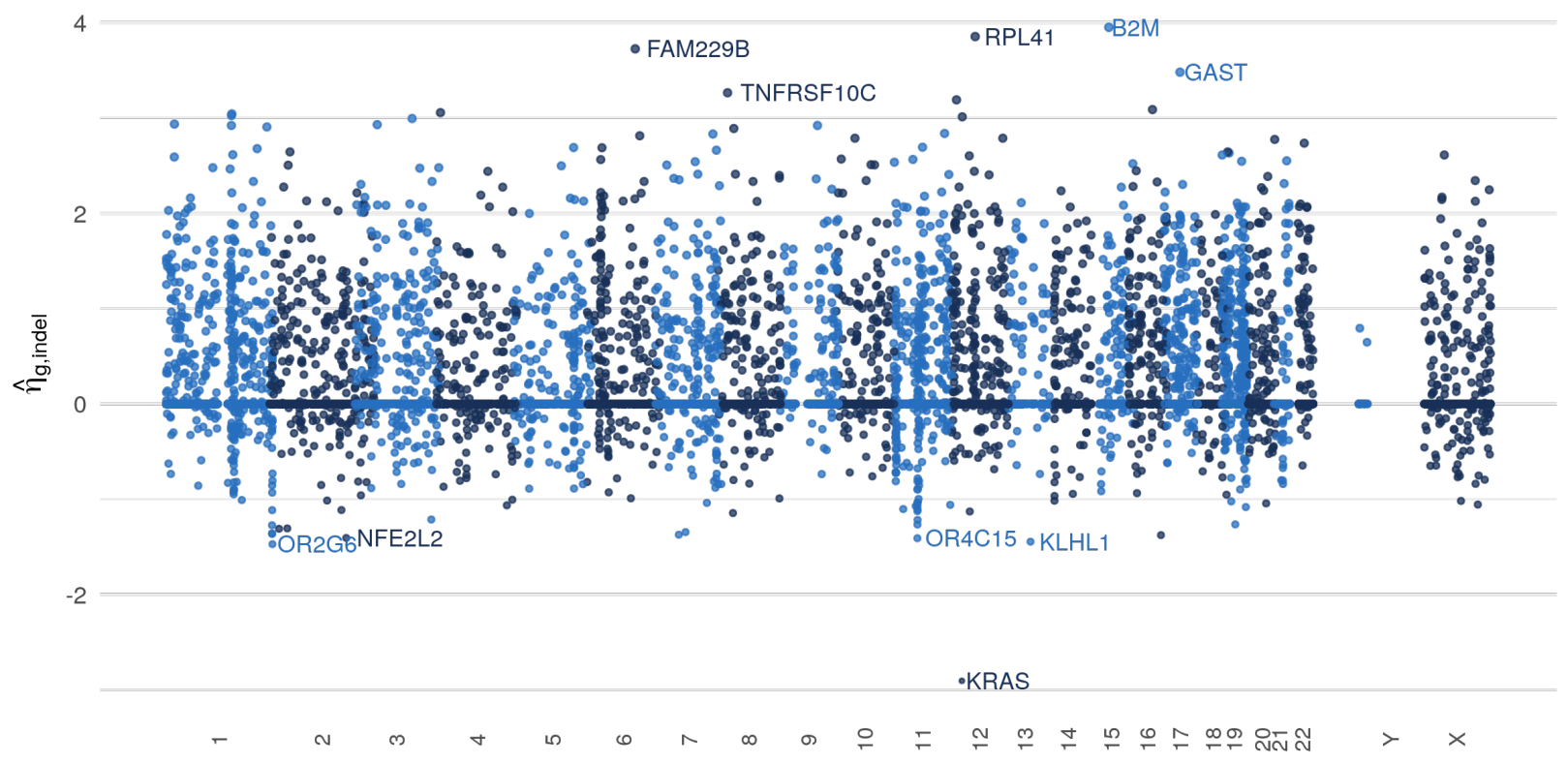

Figure 5: Manhattan plot of fitted parameters $\hat{\eta}_{g \text {,indel }}$ and their associated genes' chromosomal locations. The five largest positive and negative genes are labelled.

(i) Saturated model: the model in (2), where each observation has an associated free parameter (i.e. $\phi_{i g s}>0$ is unrestricted);

(ii) No sample-specific effects: the model in (3), with $\mu_{i}=0$ for all $i \in\{1, \ldots, n\}$;

(iii) No gene-specific effects: the model in (3), with $\lambda_{g}=\eta_{g s}=0$ for all $g \in G$ and $s \in S$;

(iv) No gene/mutation type interactions: the model in (3), with $\eta_{g s}=0$ for all $g \in G$ and $s \in S$.

In Table 1 we present the residual deviance and the residual degrees of freedom between our model and each of the models above. We see that our model is preferred over the saturated model, and all three submodels of (3).

Table 1: Model comparisons on the basis of residual deviance statistics.

\begin{tabular}{|c|c|c|c|c|}
\hline $\begin{array}{c}\text { Comparison } \\
\text { Model }\end{array}$ & $\begin{array}{c}\text { Residual } \\
\text { Deviance }(\mathrm{dev})\end{array}$ & $\begin{array}{c}\text { Residual Degrees } \\
\text { of Freedom }(\mathrm{df})\end{array}$ & $\mathrm{dev} / \mathrm{df}$ & $p$-value \\
\hline (i) & $1.43 \times 10^{6}$ & $2.74 \times 10^{7}$ & 1.00 & \\
\hline (ii) & $1.42 \times 10^{5}$ & $8.00 \times 10^{2}$ & $1.77 \times 10^{2}$ & 0.00 \\
(iii) & $1.10 \times 10^{5}$ & $1.33 \times 10^{4}$ & $8.24 \times 10^{0}$ & 0.00 \\
(iv) & $1.70 \times 10^{4}$ & $1.82 \times 10^{3}$ & $9.33 \times 10^{0}$ & 0.00 \\
\hline
\end{tabular}

\subsection{Predicting tumour mutation burden}

We now demonstrate the excellent practical performance of our procedure for estimating TMB. First it is shown that our method can indeed select gene panels of size specified by the practitioner and that good predictions can be made even with small panel sizes (i.e. $\leq$ 
$1 \mathrm{Mb})$. We then compare the performance of our proposal with state-of-the-art estimation procedures based on a number of widely used gene panels.

In order to evaluate the predictive performance of an estimator we calculate the $R^{2}$ score on the validation data as follows: given predictions of TMB, $\hat{t}_{1}, \ldots, \hat{t}_{n_{\text {val }}}$, for the observations in the validation set with true TMB values $t_{1}, \ldots, t_{n_{\text {val }}}$. Let $\bar{t}:=\frac{1}{n_{\text {val }}} \sum_{i=1}^{n_{\text {val }}} t_{i}$, and define

$$
R^{2}:=1-\frac{\sum_{i=1}^{n_{v a l}}\left(t_{i}-\hat{t}_{i}\right)^{2}}{\sum_{i=1}^{n_{v a l}}\left(t_{i}-\bar{t}\right)^{2}}
$$

Other existing works have aimed to classify tumours into two groups (high TMB, low TMB); see, for example, Büttner et al. (2019) and Wu et al. (2019b). Here we also report the estimated area under the precision-recall curve (AUPRC) for a classifier based on our estimator. We define the classifier as follows: first, in line with major clinical studies (e.g. Hellmann et al., 2018a; Ramalingam et al., 2018) the true class membership of a tumour is defined according to whether it has $t^{*}:=300$ or more exome mutations (approximately $10 \mathrm{Mut} / \mathrm{Mb})$. In the validation set, this gives $47(27.5 \%)$ tumours with high TMB and $124(72.5 \%)$ with low TMB. Now, for a cutoff $t \geq 0$, we can define a classifier by assigning a tumour to the high TMB class if its estimated TMB value is greater than or equal to $t$. For such a classifier, we have precision and recall (estimated over the validation set) given by

$$
p(t):=\frac{\sum_{i=1}^{n_{\text {val }}} \mathbb{1}_{\left\{\hat{t}_{i} \geq t, t_{i} \geq t^{*}\right\}}}{\sum_{i=1}^{n_{\text {val }}} \mathbb{1}_{\left\{\hat{t}_{i} \geq t\right\}}} \text { and } \quad r(t):=\frac{\sum_{i=1}^{n_{\text {val }}} \mathbb{1}_{\left\{\hat{t}_{i} \geq t, t_{i} \geq t^{*}\right\}}}{\sum_{i=1}^{n_{\text {val }}} \mathbb{1}_{\left\{t_{i} \geq t^{*}\right\}}}
$$

respectively. The precision-recall curve then is $\{(r(t), p(t)): t \in[0, \infty)\}$. Note that a perfect classifier achieves a AUPRC of 1 , whereas a random guess in this case would have an average AUPRC of 0.275 (the prevalence of the high TMB class).

Now recall that TMB is given by equation (1) with $\bar{S}$ being the set of all non-synonymous mutation types. Thus to estimate TMB we apply our procedure in Section 2.3 with $\bar{S}=S$, where the model parameters are estimated as described in Section 3.1. In Figure 6, we present the $R^{2}$ and AUPRC for the first-fit and refitted estimators (see (6) and (8)) as the selected panel size varies from $0 \mathrm{Mb}$ to $2 \mathrm{Mb}$ in length. We see that we obtain a more accurate prediction of TMB, both in terms of regression and classification, as the panel size increases, and that good estimation is possible even with very small panels (as low as $0.2 \mathrm{Mb}$ ). Finally, as expected, the refitted estimator slightly outperforms the first-fit estimator.

We now compare our method with state-of-the-art estimators applied to commonly used gene panels, as well as a panel selected by the proposal of Lyu et al. (2018). The three next-generation sequencing panels that we consider are chosen for their relevance to TMB. These are TST-170 (Heydt et al., 2018), Foundation One (Frampton et al., 2013) and MSKIMPACT (Cheng et al., 2015). Further, the panel selected by the approach in Lyu et al. (2018) consists of the genes that are mutated more than $10 \%$ of the time, that are less than $0.015 \mathrm{Mb}$ in length and for which the presence of a mutation in the gene is significantly associated with higher TMB values. For each panel $P \subseteq G$, we use four different methods to predict TMB:

(i) Our refitted estimator applied to the panel $P$ : we estimate TMB using $T\left(\hat{w}_{P}\right)$, where $\hat{w}_{P} \in \arg \min _{w \in W_{P}}\{f(w)\}$, and $W_{P}$ is defined in (7). 


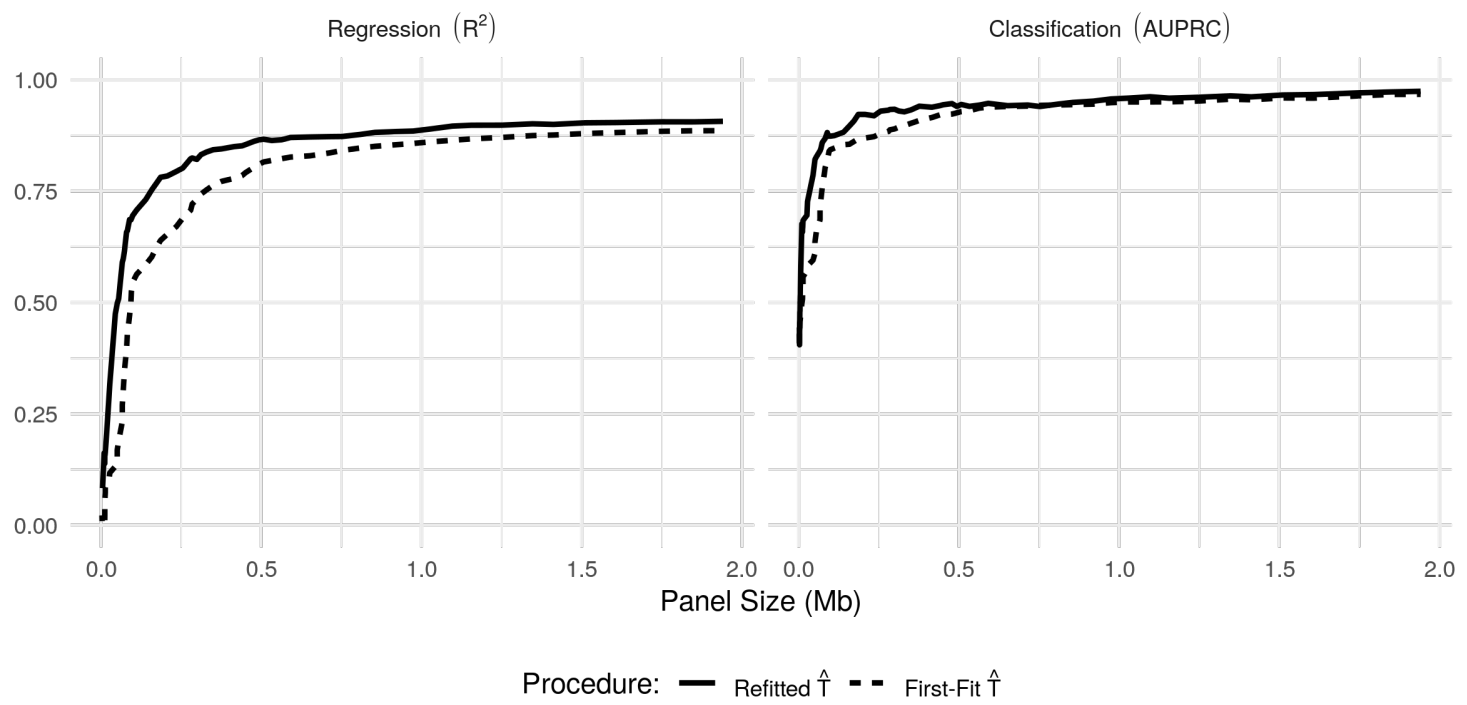

Figure 6: Performance of our first-fit and refitted estimators of TMB as the selected panel size varies. Left: $R^{2}$, Right: AUPRC.

(ii) Estimation and Classification of Tumour Mutation Burden (ecTMB): the procedure proposed by Yao et al. (2020).

(iii) A count estimator: TMB is estimated by $\frac{\ell_{G}}{\ell_{P}} \sum_{g \in P} \sum_{s \in \bar{S}} M_{0 g s}$, i.e. rescaling the mutation burden in the genes of $P$.

(iv) A linear model: we estimate TMB via ordinary least-squares linear regression of TMB against $\left\{\sum_{s \in S} M_{0 g s}: g \in P\right\}$.

The latter three comprise existing methods for estimating TMB available to practitioners. The second (ecTMB), which is based on a negative binomial model, is the state-of-the-art. The third is a standard practical procedure for the estimation of TMB from targeted gene panels. The fourth is the approach proposed by Lyu et al. (2018). The refitted estimator applied to the panel $P$ is also included here, in order to demonstrate the utility of our approach even with a prespecified panel.

We present results of these comparisons in Figure 7. First, for each of the four panels considered here, we see that our refitted estimator applied to the panel outperforms all existing approaches in terms of regression performance, and that for smaller panels we are able to improve regression accuracy even further by selecting a panel (perhaps even of smaller size) based on the training data. For instance, in comparison to predictions based on the TST-170 panel, our procedure can achieve higher $R^{2}$ with a selected panel of half the size (with $0.2 \mathrm{Mb}$ we obtain an $R^{2}$ of 0.78 ). The best available existing method based on the TST170 panel, in this case the linear estimator, has an $R^{2}$ of 0.74 . Moreover, data-driven selection of panels considerably increases the classification performance for the whole range of panel sizes considered. In particular, even for the smallest panel size shown in Figure $7(\sim 0.2 \mathrm{Mb})$, the classification performance of our method outperforms the best existing methodology applied to the MSK-IMPACT panel, despite being almost a factor of six times smaller. The full proposal of Lyu et al. (2018), which involves applying the linear regression estimate to the panel selected as described above, also performs well here. 


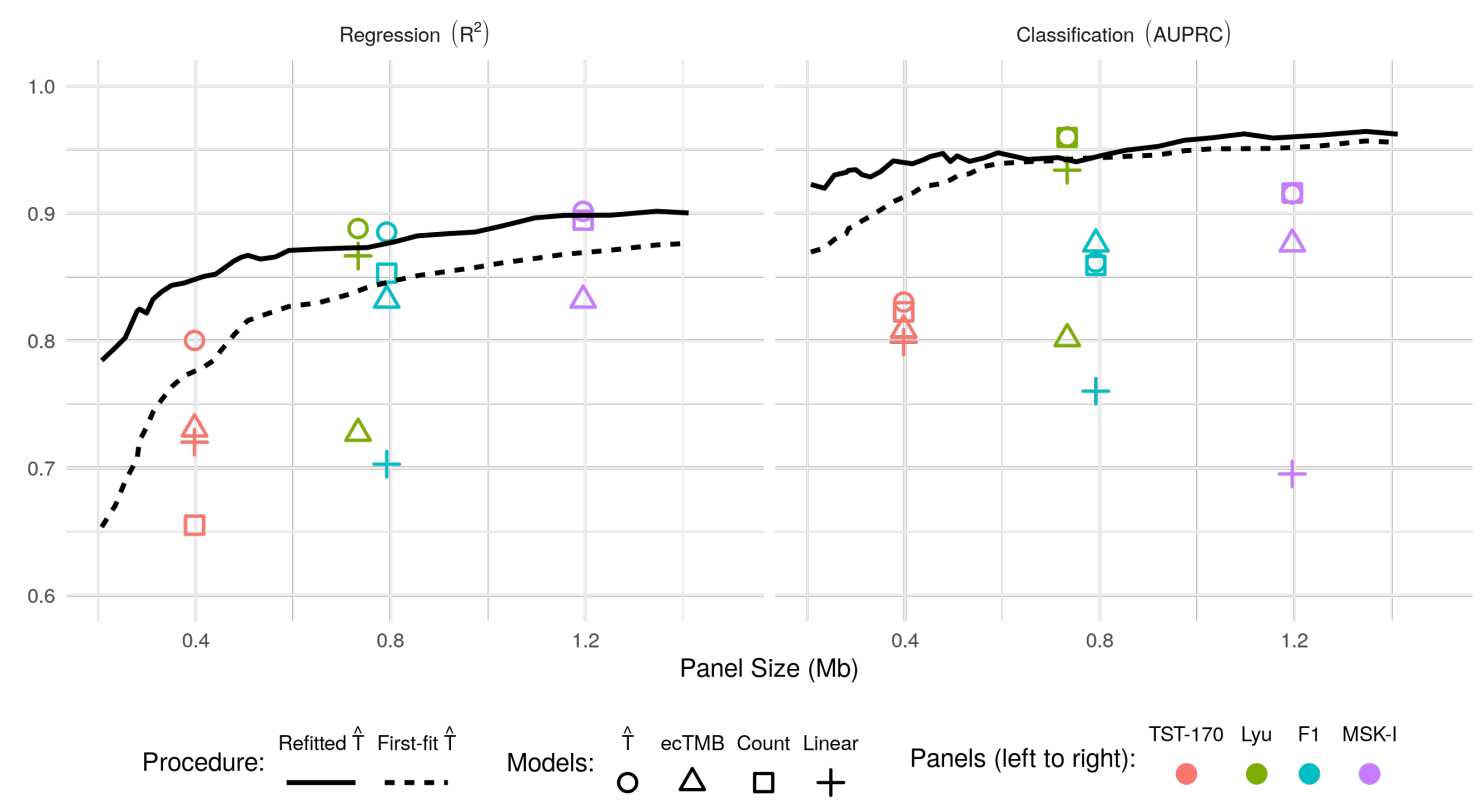

Figure 7: The performance of our TMB estimator in comparison to existing approaches. Left: $R^{2}$, Right: AUPRC.

Finally in this subsection we demonstrate the practical performance of our method using the test set, which until this point has been held out. Based on the validation results above, we take the panel of size $0.6 \mathrm{Mb}$ selected by our procedure and use our refitted estimator on that panel to predict TMB for the 173 samples in the test set. For comparison, we also present predictions from ecTMB, the count-based estimator and the linear regression estimator applied to the same panel. In Figure 8 we see that our procedure performs well; we obtain an $R^{2}$ value (on the test data) of 0.85 . The other methods have $R^{2}$ values of 0.67 (ecTMB), -36 (count) and 0.64 (linear regression). The count-based estimator here gives predictions which are reasonably well correlated to the true values of TMB but are positively biased. This is because our selection procedure tends to favour genes with higher overall mutation rates and thus a count estimator based on the highly mutated genes will overestimate the total number of mutations. We also include a red shaded region comprising all points for which heuristic 90\% prediction intervals (as described in Section 2.5) include the true TMB value. We find in this case that $93.6 \%$ of the observations in the test set fall within this region, giving valid empirical coverage.

\subsubsection{Robustness to different training datasets}

Here we investigate the robustness of our proposal to changes in the training dataset. We conduct an experiment that first involves splitting the training data set of $n=800$ observations into four disjoint datasets of 200 observations. We then retrain our model and estimator given in Sections 2.2 and 2.3 based on the four possible datasets that combine three of the four subsets. We then evaluate the predictive performance on the validation dataset similarly to in the previous subsection. The results are given in Figure 9; we see that our proposal is has very similar regression performance on the four different subsets. 


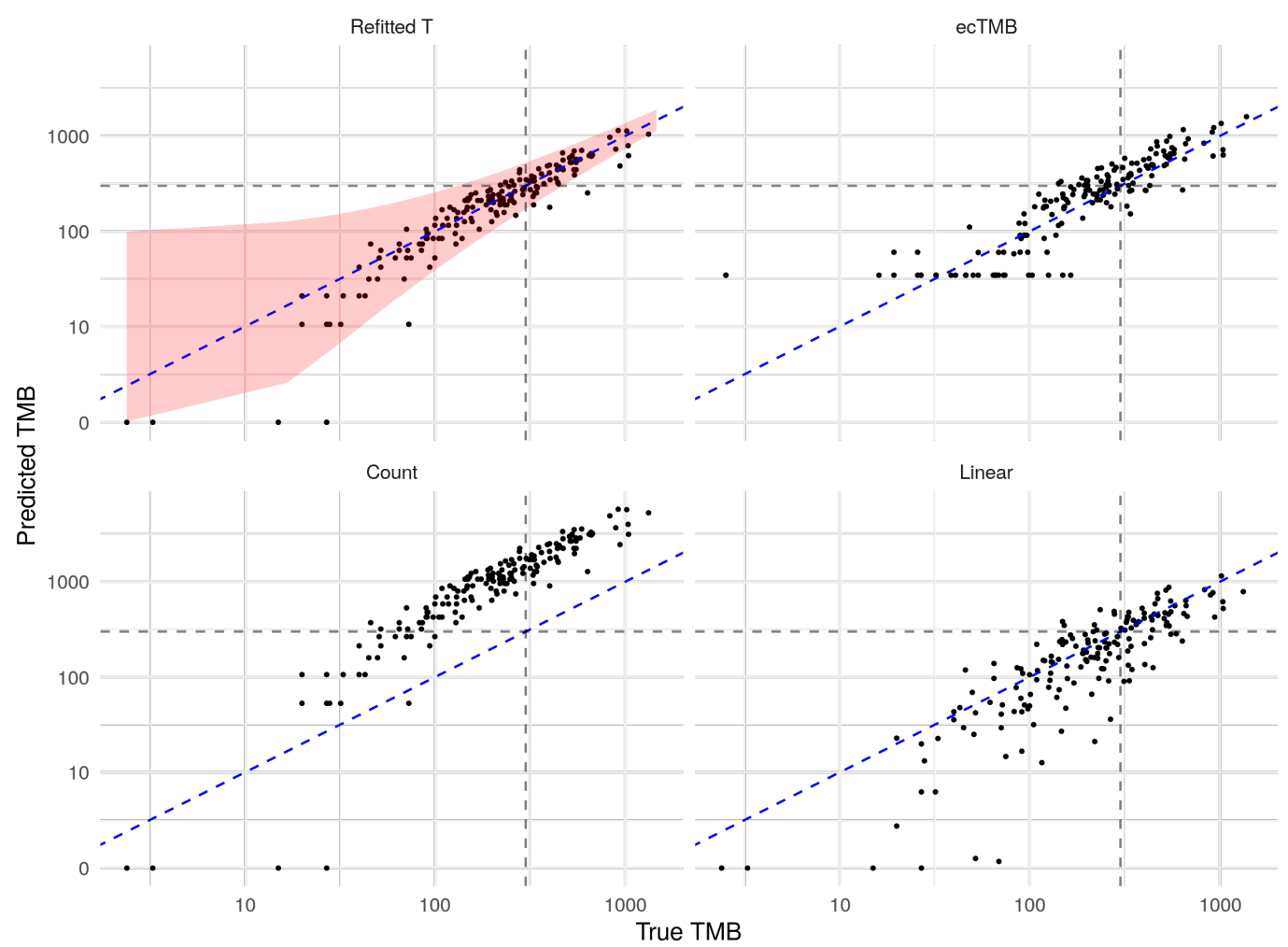

Figure 8: Prediction of TMB on the test dataset. Dashed blue (diagonal) line represents perfect prediction, and the black dashed lines indicate true and predicted TMB values of 300.

\subsection{External testing and classification of response to immunother- apy}

The aim of this section is to further test our proposed estimator of TMB by making use of two external NSCLC datasets for which the response to immunotherapy is available: Hellmann et al. (2018b), which contains 75 samples with an average TMB of 261; and Rizvi et al. (2015), which contains 34 samples with an average TMB of 258.

We first use our refitted estimator trained on the same data as in Section 3.2 to predict TMB for the samples in the new datasets using the selected panel of size $0.6 \mathrm{Mb}$. The predictions are given in Figure 10; the corresponding regression performance is $R^{2}=0.70$ across the two datasets, with a joint AUPRC for classifying tumours to high or low TMB classes of 0.91 .

These datasets also allow us to assess the practical utility of using our estimated TMB values to predict response to immunotherapy. Of the 75 samples in the Hellmann et al. (2018b) study, 37 were identified as having a Durable Clinical Benefit (Class 1) in response to immunotherapy (PD-L1+CTLA-4 blockade), and the remaining 38 were deemed to have No Benefit (Class 0). Of the 34 samples in the Rizvi et al. (2015) study, 14 were identified as having a Durable clinical benefit beyond 6 months (Class 1) in response to immunotherapy (Pembrolizumab), while the remaining 20 were deemed not to have such benefit (Class 0 ). Since the treatment and outcome definition differ between studies, we separate them for analysis of response. We construct two simple classifiers for comparison, the first assigning a sample to Class 1 if the true TMB value is greater than some threshold $t$, and the second using 


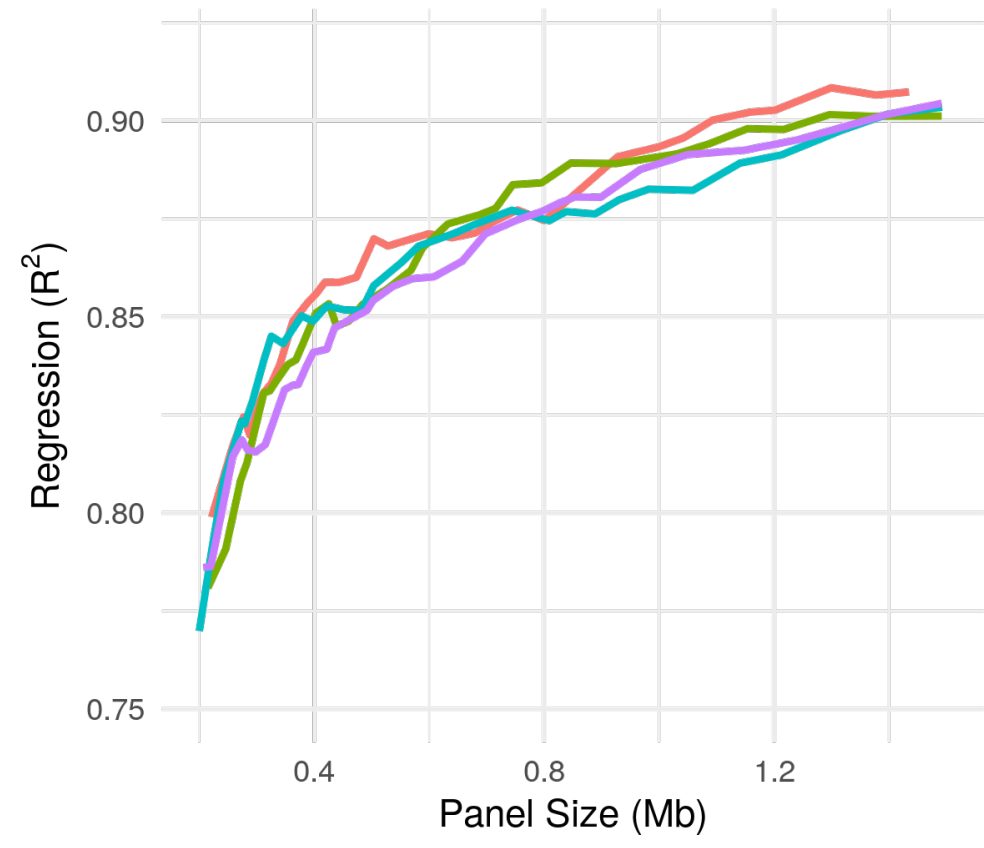

Training Subset: $-1-2-3-4$

Figure 9: The regression performance on the validation dataset for the four different training subsets of 600 observations.

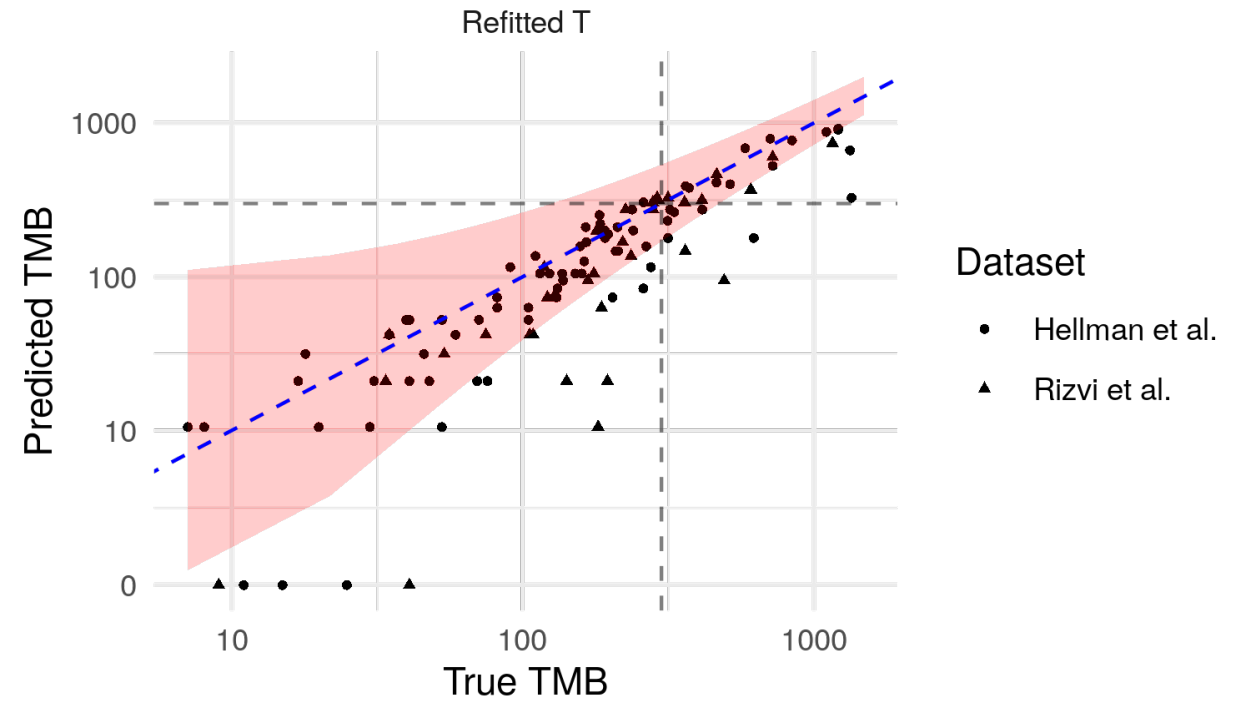

Figure 10: Performance of our model trained on the Campbell et al. (2016) dataset used to predict TMB based on the panel of size $0.6 \mathrm{Mb}$ selected by our method on the external test datasets of Hellmann et al. (2018b) and Rizvi et al. (2015) 


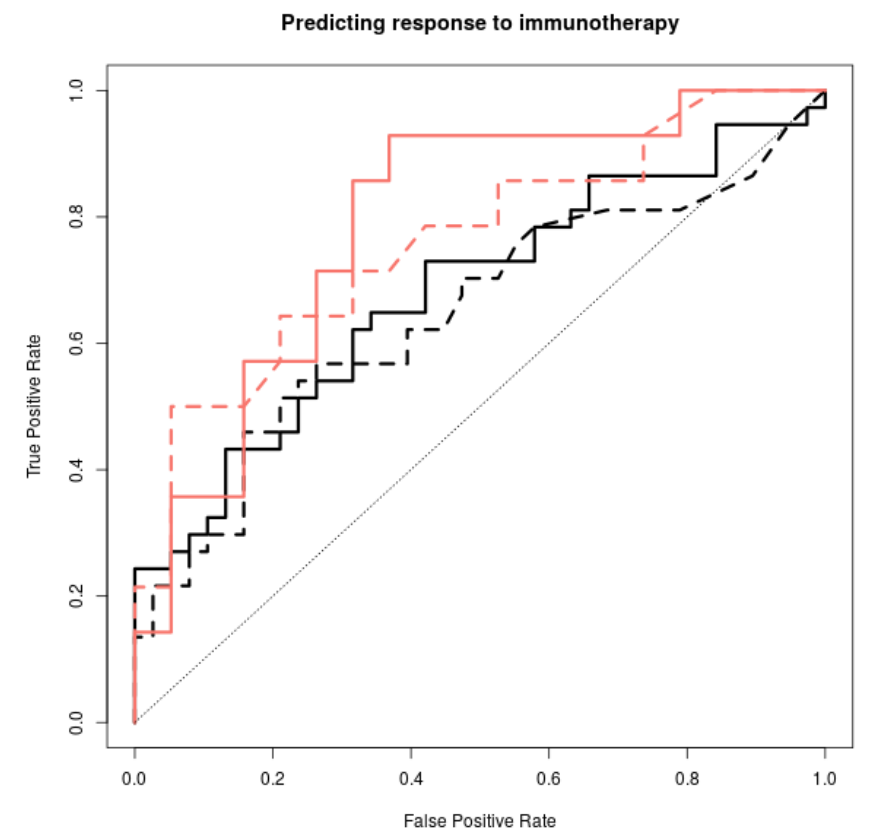

Figure 11: ROC curves for classifying the response to immunotherapy in the Hellmann et al. (2018b) (black) and Rizvi et al. (2015) (red) datasets using the true TMB values (solid) and estimated TMB values (dashed) based on the panel of size 0.6Mb selected by our method.

our estimated value of TMB in the same way. In Figure 11, we plot the Receiver Operating Characteristic (ROC) curve (that is the false positive rate against the true positive rate as the classification threshold $t$ varies). The area under the ROC curve is 0.68 for the Hellmann et al. (2018b) dataset when using the true TMB value and is 0.64 when using the estimated TMB value. The Rizvi et al. (2015) has an area under the ROC curve of 0.79 using true TMB values and 0.76 using estimated TMB values. We see that, in both cases, very little is lost in terms of predicting response to immunotherapy when using our estimated value of TMB.

\subsection{Predicting tumour indel burden}

In this section we demonstrate how our method can be used to estimate TIB. This is more challenging than estimating TMB due to the low abundance of indel mutations relative to other variant types (see Figure 2), as well as issues involved in sequencing genomic loci of repetitive nucleotide constitution (Narzisi and Schatz, 2015). Indeed, in contrast to the previous section, we are not aware of any existing methods designed to estimate TIB from targeted gene panels. We therefore investigate the performance of our method across a much wider range $(0-30 \mathrm{Mb})$ of panel sizes, and find that we are able to accurately predict TIB with larger panels. Our results also demonstrate that accurate classification of TIB status is possible even with small gene panels.

We let $S_{\text {indel }}$ be the set of all frameshift insertion and deletion mutations, and apply our method introduced in Section 2.3 with $\bar{S}=S_{\text {indel }}$. As in the previous section, we assess regression and classification performance via $R^{2}$ and AUPRC, respectively, where in this 
case tumours are separated into two classes: high TIB (10 or more indel mutations) and low TIB (otherwise). In the validation dataset, this gives 57 (33.3 tumours in the high TIB class.

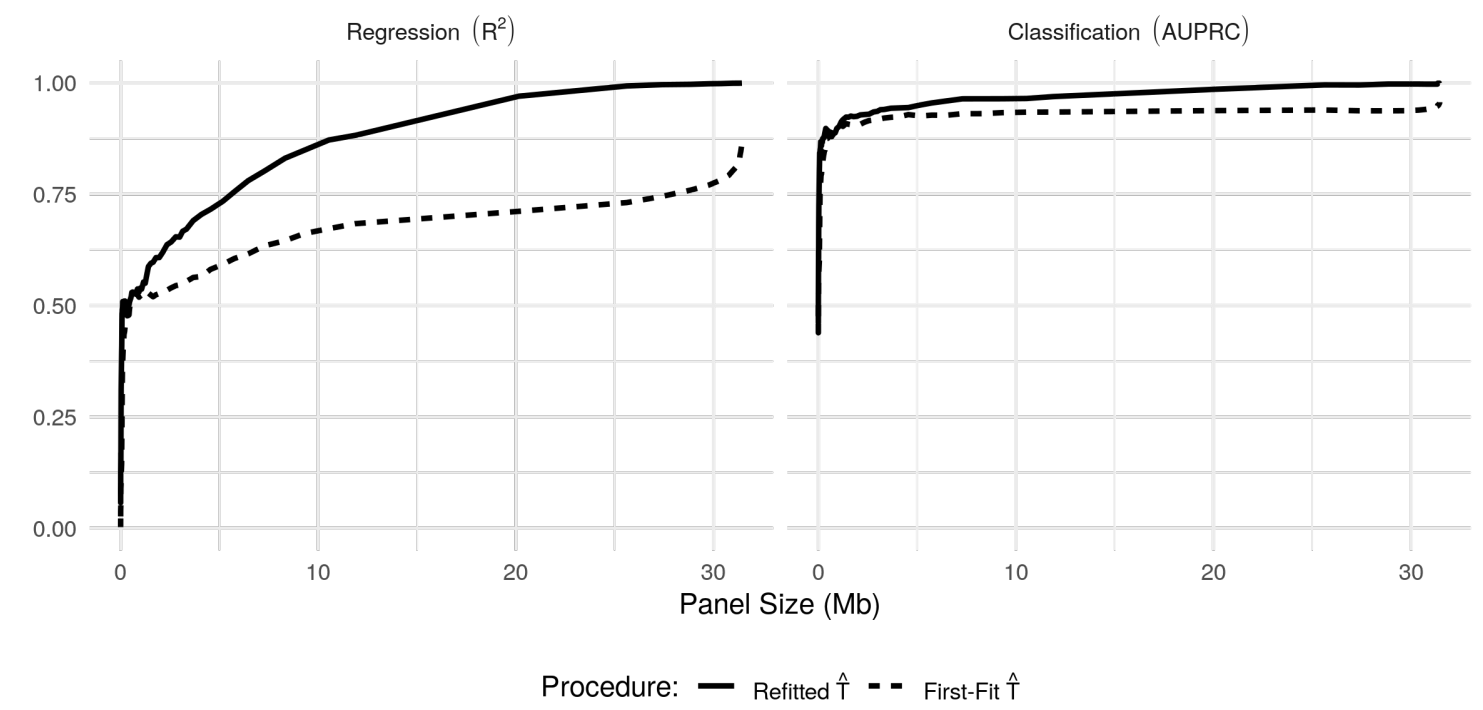

Figure 12: Performance of our first-fit and refitted estimators of TIB as the selected panel size varies. Left: $R^{2}$, Right: AUPRC.

The results are presented in Figure 12. We comment first on the regression performance: as expected, we see that the $R^{2}$ values for our first-fit and refitted estimators are much lower than what we achieved in estimating TMB. The refitted approach improves for larger panel sizes, while the first-fit estimator continues to perform relatively poorly. On the other hand, we see that the classification performance is impressive, with AUPRC values of above 0.8 for panels of less than $1 \mathrm{Mb}$ in size.

We now assess the performance on the test set of our refitted estimator of TIB applied to a selected panel of size $0.6 \mathrm{Mb}$, and we compare with a count-based estimator and linear regression estimator. We do not compare with ecTMB here, since it is designed to estimate TMB as opposed to TIB. The count-based estimator in this case scales the total number of non-synonymous mutations across the panel by the ratio of the length of the panel to that of the entire exome, and also by the relative frequency of indel mutations versus all non-synonymous mutations in the training dataset:

$$
\frac{\ell_{G}}{\ell_{P}} \frac{\sum_{i=1}^{n} \sum_{g \in G} \sum_{s \in S_{\text {indel }}} M_{i g s}}{\sum_{i=1}^{n} \sum_{g \in G} \sum_{s \in S} M_{i g s}} \sum_{g \in P} \sum_{s \in S} M_{0 g s} .
$$

In Figure 13 we present the predictions on the test set of our refitted estimator $\left(R^{2}=0.35\right)$; the count estimator $\left(R^{2}=-44\right)$; and the linear regression estimator $\left(R^{2}=-0.15\right)$. We also include (shaded in red) the set of points for which $90 \%$ prediction intervals contain the true value. In this case we find that $97.7 \%$ of test set points fall within this region.

\subsection{A panel-augmentation case study}

As discussed in Section 2.4, we may wish to include genes from a given panel, but use our methodology to augment the panel to include additional genes with goal of obtaining more 


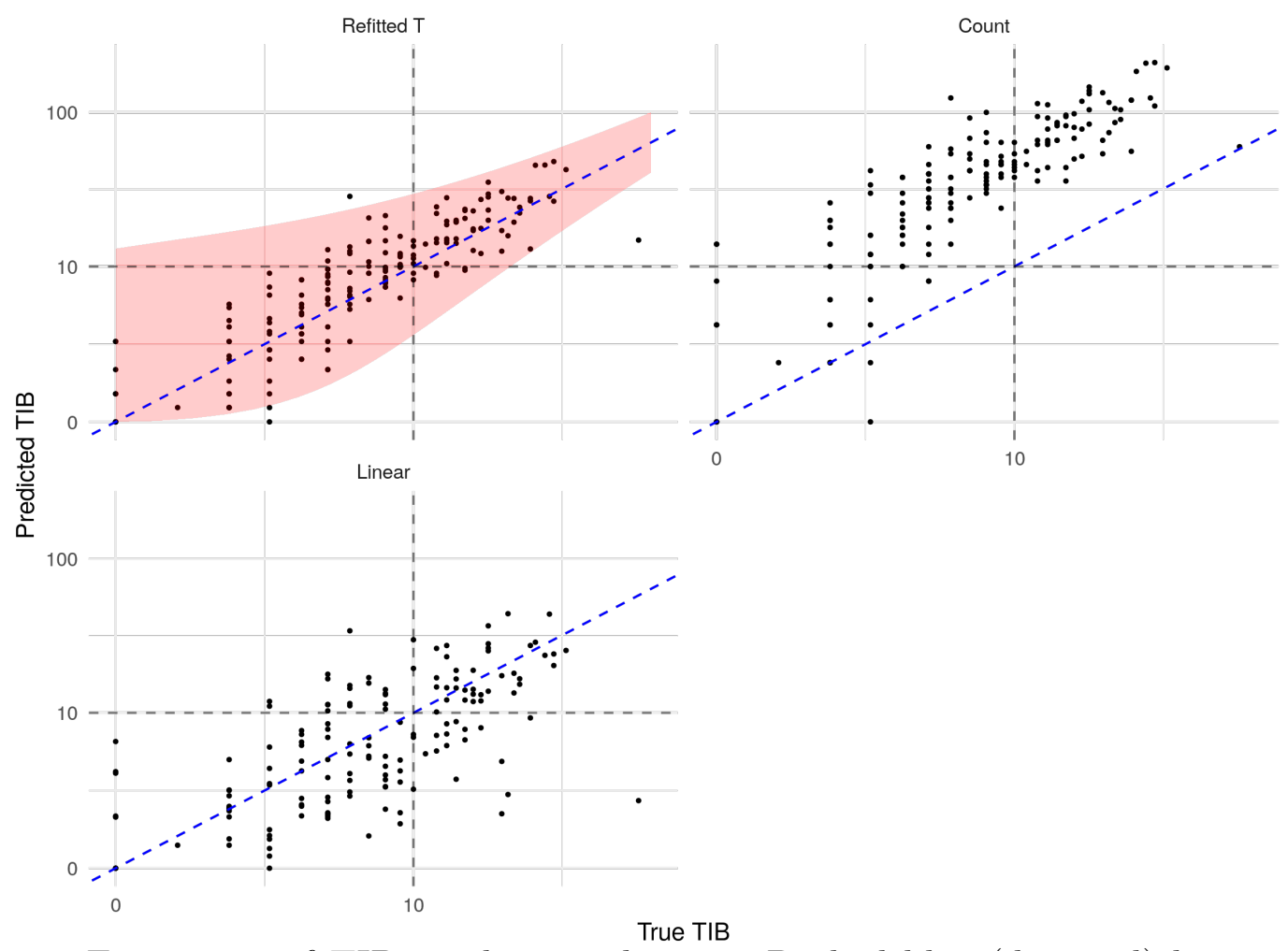

Figure 13: Estimation of TIB on the test dataset. Dashed blue (diagonal) line represents perfect prediction, and the grey dashed lines indicate true and predicted TIB values of 10 .

accurate predictions of TMB (or other biomarkers). In this section we demonstrate how this can be done starting with the TST-170 panel $(\sim 0.4 \mathrm{Mb})$ and augmenting to $0.6 \mathrm{Mb}$ in length, demonstrating impressive gains in predictive performance.

We apply the augmentation method described in Section 2.4, with $P_{0}$ taken to be the set of TST-170 genes and $Q_{0}$ to be empty. The genes added to the panel are determined by the first-fit estimator in equation (9). To evaluate the performance, we then apply the refitted estimator on the test dataset, after selecting the augmented panel of size $0.6 \mathrm{Mb}$. For comparison, we apply our refitted estimator to the TST-170 panel directly. We also present the results obtained by the other estimators described above, both before and after the panel augmentation, in Table 2. We find that by augmenting the panel we improve predictive performance with our refitted $\hat{T}$ estimator, both in terms of regression and classification. The refitted estimator provides better estimates than any other model on the augmented panel by both metrics.

Table 2: Predictive performance of models on TST-170 (0.4Mb) versus augmented TST-170 $(0.6 \mathrm{Mb})$ panels on the test set.

\begin{tabular}{|c|c|c|c|c|}
\hline \multirow{2}{*}{ Model } & \multicolumn{2}{|c|}{ Regression $\left(R^{2}\right)$} & \multicolumn{2}{c|}{ Classification (AUPRC) } \\
\cline { 2 - 5 } & TST-170 & Aug. TST-170 & TST-170 & Aug. TST-170 \\
\hline Refitted $\hat{T}$ & $\mathbf{0 . 5 8}$ & $\mathbf{0 . 8 4}$ & $\mathbf{0 . 8 4}$ & $\mathbf{0 . 9 4}$ \\
ecTMB & 0.37 & 0.51 & 0.80 & 0.88 \\
Count & 0.18 & 0.18 & $\mathbf{0 . 8 3}$ & $\mathbf{0 . 9 4}$ \\
Linear & 0.47 & 0.74 & 0.78 & 0.89 \\
\hline
\end{tabular}




\section{Further testing in other cancer types}

The aim of this section is to further demonstrate the performance of our proposed framework in a number of other cancer types. We apply our method for estimating TMB in six more cancer types, namely bladder cancer, breast cancer, colorectal cancer, melanoma, prostate cancer and renal cell cancer. For each cancer, data from two studies are used. Data from the first study is (randomly) split into a training and validation set; the training data is used to construct our estimator for a range of panel sizes, we then evaluate the predictive performance on the validation set (note that in contrast to our analysis in Section 3, we do not require a separate test set since the panel size is not selected based on the data). Further, in order to test the robustness of our approach to study effects, for each cancer type, we will also apply our fitted estimator (trained using data from the first study) to predict TMB values for tumours from the second study.

The datasets used (with training, validation and external test sample sizes in parentheses) are from the following studies:

- Bladder cancer: the bladder cancer dataset from the TCGA Pan-Cancer $\operatorname{Atlas}^{4}\left(n_{\text {train }}=\right.$ 300, $\left.n_{\mathrm{val}}=109\right)$ and Guo et al. (2013) $\left(n_{\text {test }}=99\right)$;

- Breast cancer: the breast cancer dataset from the TCGA Pan-Cancer Atlas $\left(n_{\text {train }}=\right.$ $\left.700, n_{\mathrm{val}}=300\right)$ and Kan et al. (2018) $\left(n_{\text {test }}=187\right)$;

- Colorectal cancer: Giannakis et al. (2016) $\left(n_{\text {train }}=500, n_{\text {val }}=119\right)$ and Seshagiri et al. (2012) $\left(n_{\text {test }}=72\right)$;

- Melanoma: Cancer Genome Atlas Network (2015) $\left(n_{\text {train }}=250, n_{\text {val }}=96\right)$ and Krauthammer et al. (2012) $\left(n_{\text {test }}=91\right)$;

- Prostate cancer: Armenia et al. (2018) $\left(n_{\text {train }}=700, n_{\text {val }}=312\right)$ and Kumar et al. (2016) $\left(n_{\text {test }}=141\right)$;

- Renal cell cancer: the renal cell cancer dataset from TCGA Firehose ${ }^{5}\left(n_{\text {train }}=350\right.$, $\left.n_{\mathrm{val}}=101\right)$ and Guo et al. (2011) $\left(n_{\text {test }}=98\right)$.

These datasets have a range of mutation rates, specifically the average TMB values in the training datasets are 247 (bladder cancer), 91 (breast cancer), 339 (colorectal cancer), 568 (melanoma), 63 (prostate cancer) and 77 (renal cell cancer).

In Figure 14, the black lines plot the $R^{2}$ values obtained on the internal validation set from the first study for the six cancer types as the panel size varies from $0.25 \mathrm{Mb}$ to $1.25 \mathrm{Mb}$. The blue lines show the $R^{2}$ values obtained when predicting TMB for tumours in the external test set from the second study. We see that the performance on the internal validation set is very good and broadly in line with the performance we obtained for the NSCLC dataset (with the exception of the renal cell cancer). The main factor effecting the performance appears to be the overall mutation rate; our method performs very well in cancer types with large mutation rates (colorectal cancer and melanoma), but less well in the cancers with

\footnotetext{
${ }^{4}$ data available at https://www.cbioportal.org/study/clinicalData?id=blca_tcga_pan_can_ atlas_2018

${ }^{5}$ data available at https://www.cbioportal.org/study/summary?id=kirc_tcga
} 
lower overall mutation rates (prostate and renal cell). The performance on the renal cell dataset is particularly poor due to the combination of low sample size and the low average mutation rate.

The results on the external test datasets are more mixed; there is a drop off in performance in comparison with the internal validation results for breast cancer and melanoma, but apparent improvement for prostate cancer. This highlights that study effects, such as differences in patient demographics and clinical profiles, as well as variations in sequencing technologies need to be considered carefully. In practice, one should ensure that the patients in the training data used to fit the model have similar characteristics to the intended test cohort.

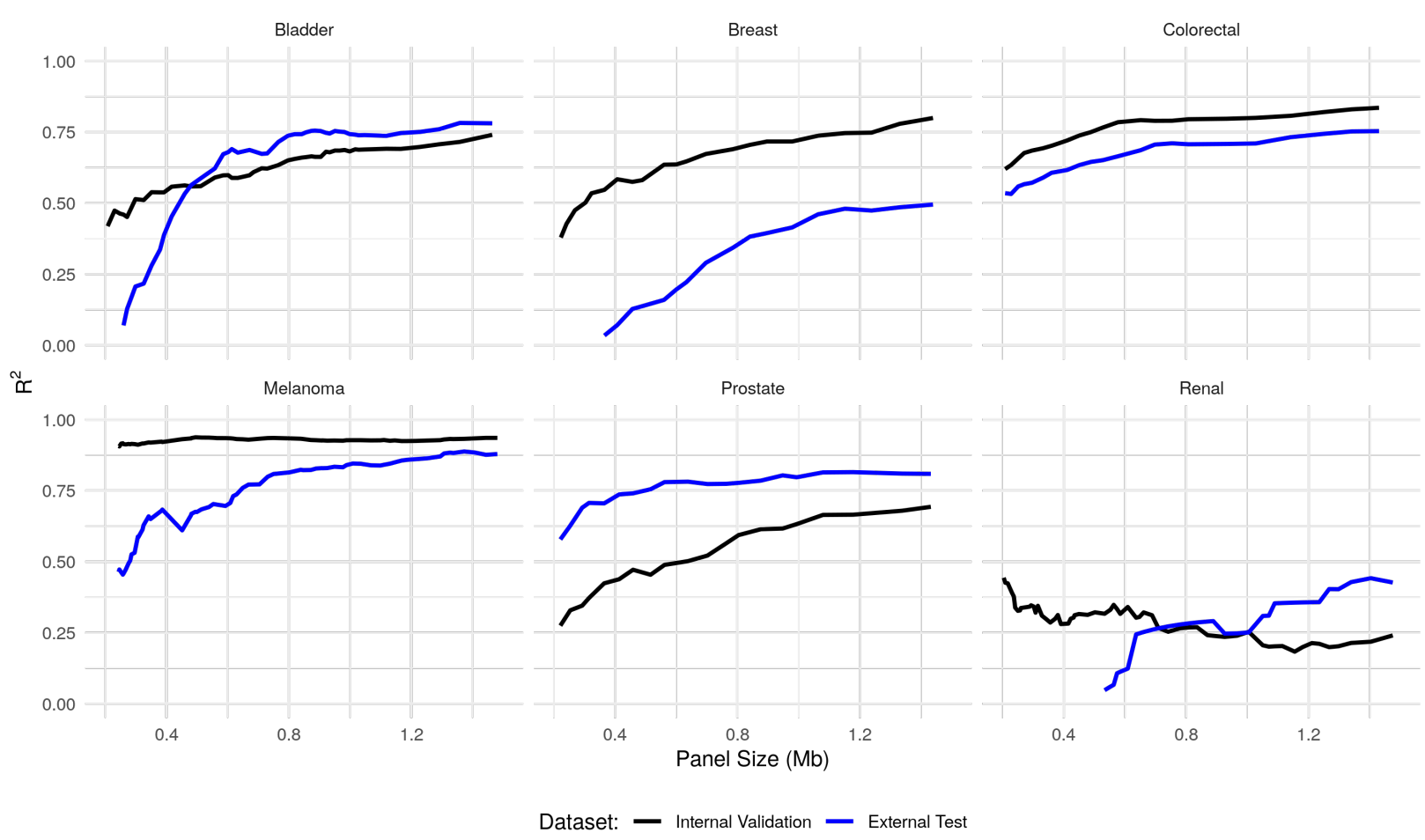

Figure 14: The performance of our refitted TMB estimator in the six further cancer types.

\section{Conclusions}

We have introduced a new data-driven framework for designing targeted gene panels which allows for cost-effective estimation of exome-wide biomarkers. Using the Non-Small Cell Lung Cancer datasets from Campbell et al. (2016), Hellmann et al. (2018b), and Rizvi et al. (2015), we have demonstrated the excellent predictive performance of our proposal for estimating Tumour Mutation Burden and Tumour Indel Burden, and shown that it outperforms the state-of-the-art procedures. We further tested the applicability and robustness of our method, by applying it to datasets on several other cancer types. Our framework can be applied to any tumour dataset containing annotated mutations, and we provide an $\mathrm{R}$ package (Bradley and Cannings, 2021) which implements the methodology. 
The main use of TMB is often to help identify patients that are more likely to respond to immunotherapy. While TMB is a good single predictor of response (Cao et al., 2019; Zhu et al., 2019), it is of course desirable to improve the predictive performance by including other factors. For instance these may include cancer type (and subtype), specific mutational signatures, aneuploidy and tumor histology, as well as other variables, such as gender, age and exogenous factors. Indeed, Litchfield et al. (2021) show that, by including markers of T-cell infiltration and other factors, a multivariate predictor of response to immunotherapy significantly improves the classification performance in comparison to using TMB alone. Nevertheless, one would certainly like to include TMB (or a closely related measure) as a factor in any classifier of response.

Our work also has the scope to help understand mutational processes. For example, the parameters of our fitted model in Section 3.1 have interesting interpretations: of the five genes highlighted in Figure 4 as having the highest mutation rates relative to the BMR, two (TP53, CDKN2A) are known tumour suppressors (Olivier et al., 2010; Foulkes et al., 1997) and $K R A S$ is an oncogene (Jancík et al., 2010). Furthermore, indel mutations in KRAS are known to be deleterious for tumour cells (Lee et al., 2018) - in our work the KRAS gene has a large negative indel-specific parameter (see Figure 5). Our methodology identifies a number of other genes with large parameter estimates. Of course, any such associations need to be carefully investigated in follow up studies.

Finally, we believe there are many ways in which our general framework can be extended. For example, it may be adapted to incorporate alternate data types (e.g. transcriptomics); we may seek to predict other features (e.g. outcomes such as survival); or we may wish to extend the method to incorporate multiple data sources (e.g. by combining data from two or more studies).

\section{Data availability}

All data used in this manuscript is publicly available. The NSCLC dataset of Campbell et al. (2016) and the Ensembl gene length dataset are available as part of our R package ICBioMark (Bradley and Cannings, 2021) - see below for more detail. The BED files for the gene panels used in Section 3.2 can be downloaded from https://github.com/cobrbra/ TargetedPanelEstimation_Paper.

\section{Code availability}

All figures and tables in this manuscript may be reproduced using the code available at https://github.com/cobrbra/TargetedPanelEstimation_Paper. We also provide an open access R package ICBioMark (Bradley and Cannings, 2021), which is available on CRAN https://cran.r-project.org. Alternatively, the package may be accessed and downloaded at https://github.com/cobrbra/ICBioMark. 


\section{Acknowledgements}

We are grateful for the constructive feedback from the anonymous reviewers, which helped to improve the paper. We gratefully acknowledge funding provided by Cambridge Cancer Genomics (CCG) through their PhD Scholarship at the University of Edinburgh. We also benefited from discussions with several individuals, including Adnan Akbar, Philip Beer, Harry Clifford, Aleksandra Jartseva, Morton, Kevin Myant, William Orchard, Nirmesh Patel and Charlotte Paterson.

\section{References}

Joshua Armenia, Stephanie A. M. Wankowicz, David Liu, Jianjiong Gao, Ritika Kundra, Ed Reznik, Walid K. Chatila, Debyani Chakravarty, G. Celine Han, Ilsa Coleman, Bruce Montgomery, Colin Pritchard, Colm Morrissey, Christopher E. Barbieri, Himisha Beltran, Andrea Sboner, Zafeiris Zafeiriou, Susana Miranda, Craig M. Bielski, Alexander V. Penson, Charlotte Tolonen, Franklin W. Huang, Dan Robinson, Yi Mi Wu, Robert Lonigro, Levi A. Garraway, Francesca Demichelis, Philip W. Kantoff, Mary-Ellen Taplin, Wassim Abida, Barry S. Taylor, Howard I. Scher, Peter S. Nelson, Johann S. de Bono, Mark A. Rubin, Charles L. Sawyers, Arul M. Chinnaiyan, PCF/SU2C International Prostate Cancer Dream Team, Nikolaus Schultz, and Eliezer M. Van Allen. The long tail of oncogenic drivers in prostate cancer. Nature Genetics, 50(5):645-651, May 2018. ISSN 1546-1718. doi: 10.1038/s41588-018-0078-z.

Hossein Borghaei, Scott Gettinger, Everett E. Vokes, Laura Q. M. Chow, Marco Angelo Burgio, Javier de Castro Carpeno, Adam Pluzanski, Oscar Arrieta, Osvaldo Arén Frontera, Rita Chiari, Charles Butts, Joanna Wójcik-Tomaszewska, Bruno Coudert, Marina Chiara Garassino, Neal Ready, Enriqueta Felip, Miriam Alonso García, David Waterhouse, Manuel Domine, Fabrice Barlesi, Scott Antonia, Markus Wohlleber, David E. Gerber, Grzegorz Czyzewicz, David R. Spigel, Lucio Crino, Wilfried Enst Erich Eberhardt, Ang Li, Sathiya Marimuthu, and Julie Brahmer. Five-Year Outcomes From the Randomized, Phase III Trials CheckMate 017 and 057: Nivolumab Versus Docetaxel in Previously Treated Non-Small-Cell Lung Cancer. Journal of Clinical Oncology: Official Journal of the American Society of Clinical Oncology, 39(7):723-733, March 2021. ISSN 1527-7755. doi: 10.1200/JCO.20.01605.

Theodor Boveri. Concerning the Origin of Malignant Tumours. Translated and annotated by Henry Harris. Journal of Cell Science, 121(Supplement 1):1-84, January 2008. ISSN 0021-9533, 1477-9137. doi: 10.1242/jcs.025742. Publisher: The Company of Biologists Ltd Section: Article.

Jacob R. Bradley and Timothy I. Cannings. ICBioMark: Data-Driven Design of Targeted Gene Panels for Estimating immunotherapy Biomarkers, February 2021. URL https: //CRAN.R-project.org/package=ICBioMark.

Elizabeth I. Buchbinder and Anupam Desai. CTLA-4 and PD-1 Pathways: Similarities, Differences, and Implications of Their Inhibition. American Journal of Clinical Oncology, 39(1):98-106, February 2016. ISSN 1537-453X. doi: 10.1097/COC.0000000000000239. 
J. Budczies, M. Allgäuer, K. Litchfield, E. Rempel, P. Christopoulos, D. Kazdal, V. Endris, M. Thomas, S. Fröhling, S. Peters, C. Swanton, P. Schirmacher, and A. Stenzinger. Optimizing panel-based tumor mutational burden (TMB) measurement. Annals of Oncology: Official Journal of the European Society for Medical Oncology, 30(9):1496-1506, September 2019. ISSN 1569-8041. doi: 10.1093/annonc/mdz205.

Reinhard Büttner, John W. Longshore, Fernando López-Ríos, Sabine Merkelbach-Bruse, Nicola Normanno, Etienne Rouleau, and Frédérique Penault-Llorca. Implementing TMB measurement in clinical practice: considerations on assay requirements. ESMO open, 4 (1):e000442, 2019. ISSN 2059-7029. doi: 10.1136/esmoopen-2018-000442.

Joshua D. Campbell, Anton Alexandrov, Jaegil Kim, Jeremiah Wala, Alice H. Berger, Chandra Sekhar Pedamallu, Sachet A. Shukla, Guangwu Guo, Angela N. Brooks, Bradley A. Murray, Marcin Imielinski, Xin Hu, Shiyun Ling, Rehan Akbani, Mara Rosenberg, Carrie Cibulskis, Aruna Ramachandran, Eric A. Collisson, David J. Kwiatkowski, Michael S. Lawrence, John N. Weinstein, Roel G. W. Verhaak, Catherine J. Wu, Peter S. Hammerman, Andrew D. Cherniack, Gad Getz, Cancer Genome Atlas Research Network, Maxim N. Artyomov, Robert Schreiber, Ramaswamy Govindan, and Matthew Meyerson. Distinct patterns of somatic genome alterations in lung adenocarcinomas and squamous cell carcinomas. Nature Genetics, 48(6):607-616, June 2016. ISSN 1546-1718. doi: 10.1038/ng.3564.

Cancer Genome Atlas Network. Genomic Classification of Cutaneous Melanoma. Cell, 161 (7):1681-1696, June 2015. ISSN 1097-4172. doi: 10.1016/j.cell.2015.05.044.

Dedong Cao, Huilin Xu, Ximing Xu, Tao Guo, and Wei Ge. High tumor mutation burden predicts better efficacy of immunotherapy: a pooled analysis of 103078 cancer patients. Oncoimmunology, 8(9):e1629258, 2019. ISSN 2162-4011. doi: 10.1080/2162402X.2019. 1629258.

Zachary R. Chalmers, Caitlin F. Connelly, David Fabrizio, Laurie Gay, Siraj M. Ali, Riley Ennis, Alexa Schrock, Brittany Campbell, Adam Shlien, Juliann Chmielecki, Franklin Huang, Yuting He, James Sun, Uri Tabori, Mark Kennedy, Daniel S. Lieber, Steven Roels, Jared White, Geoffrey A. Otto, Jeffrey S. Ross, Levi Garraway, Vincent A. Miller, Phillip J. Stephens, and Garrett M. Frampton. Analysis of 100,000 human cancer genomes reveals the landscape of tumor mutational burden. Genome Medicine, 9(1):34, April 2017. ISSN 1756-994X. doi: 10.1186/s13073-017-0424-2. URL https://doi.org/10.1186/ s13073-017-0424-2.

T. A. Chan, M. Yarchoan, E. Jaffee, C. Swanton, S. A. Quezada, A. Stenzinger, and S. Peters. Development of tumor mutation burden as an immunotherapy biomarker: utility for the oncology clinic. Annals of Oncology: Official Journal of the European Society for Medical Oncology, 30(1):44-56, January 2019. ISSN 1569-8041. doi: 10.1093/annonc/mdy 495.

Donavan T. Cheng, Talia N. Mitchell, Ahmet Zehir, Ronak H. Shah, Ryma Benayed, Aijazuddin Syed, Raghu Chandramohan, Zhen Yu Liu, Helen H. Won, Sasinya N. Scott, A. Rose Brannon, Catherine O'Reilly, Justyna Sadowska, Jacklyn Casanova, Angela Yannes, Jaclyn F. Hechtman, Jinjuan Yao, Wei Song, Dara S. Ross, Alifya Oultache, 
Snjezana Dogan, Laetitia Borsu, Meera Hameed, Khedoudja Nafa, Maria E. Arcila, Marc Ladanyi, and Michael F. Berger. Memorial Sloan Kettering-Integrated Mutation Profiling of Actionable Cancer Targets (MSK-IMPACT): A Hybridization Capture-Based Next-Generation Sequencing Clinical Assay for Solid Tumor Molecular Oncology. The Journal of molecular diagnostics: JMD, 17(3):251-264, May 2015. ISSN 1943-7811. doi: 10.1016/j.jmoldx.2014.12.006.

Laura Fancello, Sara Gandini, Pier Giuseppe Pelicci, and Luca Mazzarella. Tumor mutational burden quantification from targeted gene panels: major advancements and challenges. Journal for Immunotherapy of Cancer, 7(1):183, July 2019. ISSN 2051-1426. doi: 10.1186/s40425-019-0647-4.

Yick W. Fong, Claudia Cattoglio, and Robert Tjian. The intertwined roles of transcription and repair proteins. Molecular Cell, 52(3):291-302, November 2013. ISSN 1097-4164. doi: 10.1016/j.molcel.2013.10.018.

W. D. Foulkes, T. Y. Flanders, P. M. Pollock, and N. K. Hayward. The CDKN2A (p16) gene and human cancer. Molecular Medicine (Cambridge, Mass.), 3(1):5-20, January 1997. ISSN 1076-1551.

Garrett M. Frampton, Alex Fichtenholtz, Geoff A. Otto, Kai Wang, Sean R. Downing, Jie He, Michael Schnall-Levin, Jared White, Eric M. Sanford, Peter An, James Sun, Frank Juhn, Kristina Brennan, Kiel Iwanik, Ashley Maillet, Jamie Buell, Emily White, Mandy Zhao, Sohail Balasubramanian, Selmira Terzic, Tina Richards, Vera Banning, Lazaro Garcia, Kristen Mahoney, Zac Zwirko, Amy Donahue, Himisha Beltran, Juan Miguel Mosquera, Mark A. Rubin, Snjezana Dogan, Cyrus V. Hedvat, Michael F. Berger, Lajos Pusztai, Matthias Lechner, Chris Boshoff, Mirna Jarosz, Christine Vietz, Alex Parker, Vincent A. Miller, Jeffrey S. Ross, John Curran, Maureen T. Cronin, Philip J. Stephens, Doron Lipson, and Roman Yelensky. Development and validation of a clinical cancer genomic profiling test based on massively parallel DNA sequencing. Nature Biotechnology, 31(11): 1023-1031, November 2013. ISSN 1546-1696. doi: 10.1038/nbt.2696.

Jerome Friedman, Trevor Hastie, Rob Tibshirani, Balasubramanian Narasimhan, Kenneth Tay, Noah Simon, and Junyang Qian. glmnet: Lasso and Elastic-Net Regularized Generalized Linear Models, June 2021. URL https ://CRAN.R-project.org/package=glmnet.

Jerome H. Friedman, Trevor Hastie, and Rob Tibshirani. Regularization Paths for Generalized Linear Models via Coordinate Descent. Journal of Statistical Software, 33(1):1-22, February 2010. ISSN 1548-7660. doi: 10.18637/jss.v033.i01.

David R. Gandara, Sarah M. Paul, Marcin Kowanetz, Erica Schleifman, Wei Zou, Yan Li, Achim Rittmeyer, Louis Fehrenbacher, Geoff Otto, Christine Malboeuf, Daniel S. Lieber, Doron Lipson, Jacob Silterra, Lukas Amler, Todd Riehl, Craig A. Cummings, Priti S. Hegde, Alan Sandler, Marcus Ballinger, David Fabrizio, Tony Mok, and David S. Shames. Blood-based tumor mutational burden as a predictor of clinical benefit in nonsmall-cell lung cancer patients treated with atezolizumab. Nature Medicine, 24(9):14411448, September 2018. ISSN 1546-170X. doi: 10.1038/s41591-018-0134-3. 
Marios Giannakis, Xinmeng Jasmine Mu, Sachet A. Shukla, Zhi Rong Qian, Ofir Cohen, Reiko Nishihara, Samira Bahl, Yin Cao, Ali Amin-Mansour, Mai Yamauchi, Yasutaka Sukawa, Chip Stewart, Mara Rosenberg, Kosuke Mima, Kentaro Inamura, Katsuhiko Nosho, Jonathan A. Nowak, Michael S. Lawrence, Edward L. Giovannucci, Andrew T. Chan, Kimmie Ng, Jeffrey A. Meyerhardt, Eliezer M. Van Allen, Gad Getz, Stacey B. Gabriel, Eric S. Lander, Catherine J. Wu, Charles S. Fuchs, Shuji Ogino, and Levi A. Garraway. Genomic Correlates of Immune-Cell Infiltrates in Colorectal Carcinoma. Cell Reports, 15(4):857-865, April 2016. ISSN 2211-1247. doi: 10.1016/j.celrep.2016.03.075.

Mahdi Golkaram, Chen Zhao, Kristina Kruglyak, Shile Zhang, and Sven Bilke. The interplay between cancer type, panel size and tumor mutational burden threshold in patient selection for cancer immunotherapy. PLOS Computational Biology, 16(11):e1008332, November 2020. ISSN 1553-7358. doi: 10.1371/journal.pcbi.1008332. URL https://journals . plos.org/ploscompbiol/article?id=10.1371/journal. pcbi. 1008332.

Gail Gong and Francisco J. Samaniego. Pseudo Maximum Likelihood Estimation: Theory and Applications. The Annals of Statistics, 9(4):861-869, 1981. ISSN 0090-5364.

Guangwu Guo, Yaoting Gui, Shengjie Gao, Aifa Tang, Xueda Hu, Yi Huang, Wenlong Jia, Zesong Li, Minghui He, Liang Sun, Pengfei Song, Xiaojuan Sun, Xiaokun Zhao, Sangming Yang, Chaozhao Liang, Shengqing Wan, Fangjian Zhou, Chao Chen, Jialou Zhu, Xianxin Li, Minghan Jian, Liang Zhou, Rui Ye, Peide Huang, Jing Chen, Tao Jiang, Xiao Liu, Yong Wang, Jing Zou, Zhimao Jiang, Renhua Wu, Song Wu, Fan Fan, Zhongfu Zhang, Lin Liu, Ruilin Yang, Xingwang Liu, Haibo Wu, Weihua Yin, Xia Zhao, Yuchen Liu, Huanhuan Peng, Binghua Jiang, Qingxin Feng, Cailing Li, Jun Xie, Jingxiao Lu, Karsten Kristiansen, Yingrui Li, Xiuqing Zhang, Songgang Li, Jian Wang, Huanming Yang, Zhiming Cai, and Jun Wang. Frequent mutations of genes encoding ubiquitin-mediated proteolysis pathway components in clear cell renal cell carcinoma. Nature Genetics, 44(1):17-19, December 2011. ISSN 1546-1718. doi: 10.1038/ng.1014.

Guangwu Guo, Xiaojuan Sun, Chao Chen, Song Wu, Peide Huang, Zesong Li, Michael Dean, Yi Huang, Wenlong Jia, Quan Zhou, Aifa Tang, Zuoquan Yang, Xianxin Li, Pengfei Song, Xiaokun Zhao, Rui Ye, Shiqiang Zhang, Zhao Lin, Mingfu Qi, Shengqing Wan, Liangfu Xie, Fan Fan, Michael L. Nickerson, Xiangjun Zou, Xueda Hu, Li Xing, Zhaojie Lv, Hongbin Mei, Shengjie Gao, Chaozhao Liang, Zhibo Gao, Jingxiao Lu, Yuan Yu, Chunxiao Liu, Lin Li, Xiaodong Fang, Zhimao Jiang, Jie Yang, Cailing Li, Xin Zhao, Jing Chen, Fang Zhang, Yongqi Lai, Zheguang Lin, Fangjian Zhou, Hao Chen, Hsiao Chang Chan, Shirley Tsang, Dan Theodorescu, Yingrui Li, Xiuqing Zhang, Jian Wang, Huanming Yang, Yaoting Gui, Jun Wang, and Zhiming Cai. Whole-genome and whole-exome sequencing of bladder cancer identifies frequent alterations in genes involved in sister chromatid cohesion and segregation. Nature Genetics, 45(12):1459-1463, December 2013. ISSN 1546-1718. doi: $10.1038 / \mathrm{ng} .2798$.

Wenbing Guo, Yelin Fu, Liangliang Jin, Kai Song, Ruihan Yu, Tianhao Li, Lishuang Qi, Yunyan Gu, Wenyuan Zhao, and Zheng Guo. An Exon Signature to Estimate the Tumor Mutational Burden of Right-sided Colon Cancer Patients. Journal of Cancer, 11(4):883892, January 2020. ISSN 1837-9664. doi: 10.7150/jca.34363. URL https://www.ncbi. nlm.nih.gov/pmc/articles/PMC6959028/. 
Matthew D. Hellmann, Tudor-Eliade Ciuleanu, Adam Pluzanski, Jong Seok Lee, Gregory A. Otterson, Clarisse Audigier-Valette, Elisa Minenza, Helena Linardou, Sjaak Burgers, Pamela Salman, Hossein Borghaei, Suresh S. Ramalingam, Julie Brahmer, Martin Reck, Kenneth J. O'Byrne, William J. Geese, George Green, Han Chang, Joseph Szustakowski, Prabhu Bhagavatheeswaran, Diane Healey, Yali Fu, Faith Nathan, and Luis Paz-Ares. Nivolumab plus Ipilimumab in Lung Cancer with a High Tumor Mutational Burden. The New England Journal of Medicine, 378(22):2093-2104, May 2018a. ISSN 1533-4406. doi: 10.1056/NEJMoa1801946.

Matthew D. Hellmann, Tavi Nathanson, Hira Rizvi, Benjamin C. Creelan, Francisco Sanchez-Vega, Arun Ahuja, Ai Ni, Jacki B. Novik, Levi M. B. Mangarin, Mohsen AbuAkeel, Cailian Liu, Jennifer L. Sauter, Natasha Rekhtman, Eliza Chang, Margaret K. Callahan, Jamie E. Chaft, Martin H. Voss, Megan Tenet, Xue-Mei Li, Kelly Covello, Andrea Renninger, Patrik Vitazka, William J. Geese, Hossein Borghaei, Charles M. Rudin, Scott J. Antonia, Charles Swanton, Jeff Hammerbacher, Taha Merghoub, Nicholas McGranahan, Alexandra Snyder, and Jedd D. Wolchok. Genomic Features of Response to Combination Immunotherapy in Patients with Advanced Non-Small-Cell Lung Cancer. Cancer Cell, 33(5):843-852.e4, May 2018b. ISSN 1878-3686. doi: 10.1016/j.ccell.2018.03. 018.

C. Heydt, R. Pappesch, K. Stecker, J. Neumann, R. Buettner, and S. MerkelbachBruse. Evaluation of the TruSight Tumor 170 (TST170) assay and its value in clinical research. Annals of Oncology, 29:vi7-vi8, September 2018. ISSN 0923-7534, 15698041. doi: 10.1093/annonc/mdy318.003. URL https://www. annalsof oncology.org/ article/S0923-7534(19)32372-5/abstract.

Y. Ishida, Y. Agata, K. Shibahara, and T. Honjo. Induced expression of PD-1, a novel member of the immunoglobulin gene superfamily, upon programmed cell death. The EMBO journal, 11(11):3887-3895, November 1992. ISSN 0261-4189.

Sylwia Jancík, Jirí Drábek, Danuta Radzioch, and Marián Hajdúch. Clinical relevance of KRAS in human cancers. Journal of Biomedicine \&3 Biotechnology, 2010:150960, 2010. ISSN 1110-7251. doi: 10.1155/2010/150960.

Zhengyan Kan, Ying Ding, Jinho Kim, Hae Hyun Jung, Woosung Chung, Samir Lal, Soonweng Cho, Julio Fernandez-Banet, Se Kyung Lee, Seok Won Kim, Jeong Eon Lee, YoonLa Choi, Shibing Deng, Ji-Yeon Kim, Jin Seok Ahn, Ying Sha, Xinmeng Jasmine Mu, Jae-Yong Nam, Young-Hyuck Im, Soohyeon Lee, Woong-Yang Park, Seok Jin Nam, and Yeon Hee Park. Multi-omics profiling of younger Asian breast cancers reveals distinctive molecular signatures. Nature Communications, 9(1):1725, April 2018. ISSN 2041-1723. doi: 10.1038/s41467-018-04129-4.

Michael Krauthammer, Yong Kong, Byung Hak Ha, Perry Evans, Antonella Bacchiocchi, James P. McCusker, Elaine Cheng, Matthew J. Davis, Gerald Goh, Murim Choi, Stephan Ariyan, Deepak Narayan, Ken Dutton-Regester, Ana Capatana, Edna C. Holman, Marcus Bosenberg, Mario Sznol, Harriet M. Kluger, Douglas E. Brash, David F. Stern, Miguel A. Materin, Roger S. Lo, Shrikant Mane, Shuangge Ma, Kenneth K. Kidd, Nicholas K. Hayward, Richard P. Lifton, Joseph Schlessinger, Titus J. Boggon, and Ruth Halaban. Exome 
sequencing identifies recurrent somatic RAC1 mutations in melanoma. Nature Genetics, 44(9):1006-1014, September 2012. ISSN 1546-1718. doi: 10.1038/ng.2359.

Akash Kumar, Ilsa Coleman, Colm Morrissey, Xiaotun Zhang, Lawrence D. True, Roman Gulati, Ruth Etzioni, Hamid Bolouri, Bruce Montgomery, Thomas White, Jared M. Lucas, Lisha G. Brown, Ruth F. Dumpit, Navonil DeSarkar, Celestia Higano, Evan Y. Yu, Roger Coleman, Nikolaus Schultz, Min Fang, Paul H. Lange, Jay Shendure, Robert L. Vessella, and Peter S. Nelson. Substantial interindividual and limited intraindividual genomic diversity among tumors from men with metastatic prostate cancer. Nature Medicine, 22 (4):369-378, April 2016. ISSN 1546-170X. doi: 10.1038/nm.4053.

D. R. Leach, M. F. Krummel, and J. P. Allison. Enhancement of antitumor immunity by CTLA-4 blockade. Science (New York, N.Y.), 271(5256):1734-1736, March 1996. ISSN 0036-8075. doi: 10.1126/science.271.5256.1734.

Heidi Ledford, Holly Else, and Matthew Warren. Cancer immunologists scoop medicine Nobel prize. Nature, 562(7725):20-21, October 2018. doi: 10.1038/d41586-018-06751-0. Number: 7725 Publisher: Nature Publishing Group.

Wookjae Lee, Joon Ho Lee, Soyeong Jun, Ji Hyun Lee, and Duhee Bang. Selective targeting of KRAS oncogenic alleles by CRISPR/Cas9 inhibits proliferation of cancer cells. Scientific Reports, 8(1):11879, August 2018. ISSN 2045-2322. doi: 10.1038/s41598-018-30205-2.

Kevin Litchfield, James L. Reading, Clare Puttick, Krupa Thakkar, Chris Abbosh, Robert Bentham, Thomas B. K. Watkins, Rachel Rosenthal, Dhruva Biswas, Andrew Rowan, Emilia Lim, Maise Al Bakir, Virginia Turati, José Afonso Guerra-Assunção, Lucia Conde, Andrew J. S. Furness, Sunil Kumar Saini, Sine R. Hadrup, Javier Herrero, Se-Hoon Lee, Peter Van Loo, Tariq Enver, James Larkin, Matthew D. Hellmann, Samra Turajlic, Sergio A. Quezada, Nicholas McGranahan, and Charles Swanton. Meta-analysis of tumorand $\mathrm{T}$ cell-intrinsic mechanisms of sensitization to checkpoint inhibition. Cell, 184(3): 596-614.e14, February 2021. ISSN 1097-4172. doi: 10.1016/j.cell.2021.01.002.

Guan-Yi Lyu, Yu-Hsuan Yeh, Yi-Chen Yeh, and Yu-Chao Wang. Mutation load estimation model as a predictor of the response to cancer immunotherapy. NPJ genomic medicine, 3:12, 2018. ISSN 2056-7944. doi: 10.1038/s41525-018-0051-x.

Kateryna D. Makova and Ross C. Hardison. The effects of chromatin organization on variation in mutation rates in the genome. Nature Reviews. Genetics, 16(4):213-223, April 2015. ISSN 1471-0064. doi: 10.1038/nrg3890.

Tom Michoel. Natural coordinate descent algorithm for L1-penalised regression in generalised linear models. Computational Statistics \& Data Analysis, 97:60-70, May 2016. ISSN 01679473. doi: 10.1016/j.csda.2015.11.009.

Giuseppe Narzisi and Michael C. Schatz. The Challenge of Small-Scale Repeats for Indel Discovery. Frontiers in Bioengineering and Biotechnology, 3, January 2015. ISSN 22964185. doi: 10.3389/fbioe.2015.00008. 
Theodore S. Nowicki, Siwen Hu-Lieskovan, and Antoni Ribas. Mechanisms of Resistance to PD-1 and PD-L1 blockade. Cancer journal (Sudbury, Mass.), 24(1):47-53, 2018. ISSN 1528-9117. doi: 10.1097/PPO.0000000000000303. URL https://www.ncbi.nlm.nih. gov/pmc/articles/PMC5785093/.

Magali Olivier, Monica Hollstein, and Pierre Hainaut. TP53 Mutations in Human Cancers: Origins, Consequences, and Clinical Use. Cold Spring Harbor Perspectives in Biology, 2 (1), January 2010. ISSN 1943-0264. doi: 10.1101/cshperspect.a001008.

Drew M. Pardoll. The blockade of immune checkpoints in cancer immunotherapy. Nature reviews. Cancer, 12(4):252-264, March 2012. ISSN 1474-175X. doi: 10.1038/nrc3239.

Suresh S. Ramalingam, Matthew D. Hellmann, Mark M. Awad, Hossein Borghaei, Justin Gainor, Julie Brahmer, David R. Spigel, Martin Reck, Kenneth J. O'Byrne, Luis Paz-Ares, Kim Zerba, Xuemei Li, William J. Geese, George Green, Brian Lestini, Joseph D. Szustakowski, Han Chang, and Neal Ready. Tumor mutational burden (TMB) as a biomarker for clinical benefit from dual immune checkpoint blockade with nivolumab (nivo) + ipilimumab (ipi) in first-line (1L) non-small cell lung cancer (NSCLC): identification of TMB cutoff from CheckMate 568. Cancer Research, 78(13 Supplement):CT078-CT078, July 2018. ISSN 0008-5472, 1538-7445. doi: 10.1158/1538-7445.AM2018-CT078. URL https://cancerres . aacrjournals .org/content/78/13_Supplement/CT078.

Naiyer A. Rizvi, Matthew D. Hellmann, Alexandra Snyder, Pia Kvistborg, Vladimir Makarov, Jonathan J. Havel, William Lee, Jianda Yuan, Phillip Wong, Teresa S. Ho, Martin L. Miller, Natasha Rekhtman, Andre L. Moreira, Fawzia Ibrahim, Cameron Bruggeman, Billel Gasmi, Roberta Zappasodi, Yuka Maeda, Chris Sander, Edward B. Garon, Taha Merghoub, Jedd D. Wolchok, Ton N. Schumacher, and Timothy A. Chan. Mutational landscape determines sensitivity to PD-1 blockade in non-small cell lung cancer. Science (New York, N.Y.), 348(6230):124-128, April 2015. ISSN 0036-8075. doi: 10.1126/ science.aaa1348. URL https://www.ncbi.nlm.nih.gov/pmc/articles/PMC4993154/.

Caroline Robert. A decade of immune-checkpoint inhibitors in cancer therapy. Nature Communications, 11(1):3801, July 2020. ISSN 2041-1723. doi: 10.1038/s41467-020-17670-y.

Volker Roth and Bernd Fischer. The Group-Lasso for generalized linear models: uniqueness of solutions and efficient algorithms. In Proceedings of the 25th international conference on Machine learning, ICML '08, pages 848-855, New York, NY, USA, July 2008. Association for Computing Machinery. ISBN 9781605582054. doi: 10.1145/1390156.1390263.

Andrea Sboner, Xinmeng Jasmine Mu, Dov Greenbaum, Raymond K. Auerbach, and Mark B. Gerstein. The real cost of sequencing: higher than you think! Genome Biology, 12(8):125, August 2011. ISSN 1474-760X. doi: 10.1186/gb-2011-12-8-125. URL https://doi .org/10.1186/gb-2011-12-8-125.

Somasekar Seshagiri, Eric W. Stawiski, Steffen Durinck, Zora Modrusan, Elaine E. Storm, Caitlin B. Conboy, Subhra Chaudhuri, Yinghui Guan, Vasantharajan Janakiraman, Bijay S. Jaiswal, Joseph Guillory, Connie Ha, Gerrit J. P. Dijkgraaf, Jeremy Stinson, Florian Gnad, Melanie A. Huntley, Jeremiah D. Degenhardt, Peter M. Haverty, Richard Bourgon, Weiru Wang, Hartmut Koeppen, Robert Gentleman, Timothy K. Starr, Zemin Zhang, 
David A. Largaespada, Thomas D. Wu, and Frederic J. de Sauvage. Recurrent R-spondin fusions in colon cancer. Nature, 488(7413):660-664, August 2012. ISSN 1476-4687. doi: 10.1038/nature11282.

Samra Turajlic, Kevin Litchfield, Hang Xu, Rachel Rosenthal, Nicholas McGranahan, James L. Reading, Yien Ning S. Wong, Andrew Rowan, Nnennaya Kanu, Maise Al Bakir, Tim Chambers, Roberto Salgado, Peter Savas, Sherene Loi, Nicolai J. Birkbak, Laurent Sansregret, Martin Gore, James Larkin, Sergio A. Quezada, and Charles Swanton. Insertion-and-deletion-derived tumour-specific neoantigens and the immunogenic phenotype: a pan-cancer analysis. The Lancet. Oncology, 18(8):1009-1021, August 2017. ISSN 1474-5488. doi: 10.1016/S1470-2045(17)30516-8.

Hao-Xiang Wu, Zi-Xian Wang, Qi Zhao, Dong-Liang Chen, Ming-Ming He, Lu-Ping Yang, Ying-Nan Wang, Ying Jin, Chao Ren, Hui-Yan Luo, Zhi-Qiang Wang, and Feng Wang. Tumor mutational and indel burden: a systematic pan-cancer evaluation as prognostic biomarkers. Annals of Translational Medicine, 7(22):640, November 2019a. ISSN 23055839. doi: $10.21037 /$ atm.2019.10.116.

Hao-Xiang Wu, Zi-Xian Wang, Qi Zhao, Feng Wang, and Rui-Hua Xu. Designing gene panels for tumor mutational burden estimation: the need to shift from 'correlation' to 'accuracy'. Journal for Immunotherapy of Cancer, 7(1):206, August 2019b. ISSN 2051-1426. doi: 10.1186/s40425-019-0681-2.

Yi Yang and Hui Zou. A fast unified algorithm for solving group-lasso penalize learning problems. Statistics and Computing, 25(6):1129-1141, November 2015. ISSN 1573-1375. doi: 10.1007/s11222-014-9498-5.

Yi Yang, Hui Zou, and Sahir Bhatnagar. gglasso: Group Lasso Penalized Learning Using a Unified BMD Algorithm, March 2020. URL https://CRAN.R-project.org/package= gglasso.

Lijing Yao, Yao Fu, Marghoob Mohiyuddin, and Hugo Y. K. Lam. ecTMB: a robust method to estimate and classify tumor mutational burden. Scientific Reports, 10(1):4983, March 2020. ISSN 2045-2322. doi: 10.1038/s41598-020-61575-1.

Andrew D. Yates, Premanand Achuthan, Wasiu Akanni, James Allen, Jamie Allen, Jorge Alvarez-Jarreta, M. Ridwan Amode, Irina M. Armean, Andrey G. Azov, Ruth Bennett, Jyothish Bhai, Konstantinos Billis, Sanjay Boddu, José Carlos Marugán, Carla Cummins, Claire Davidson, Kamalkumar Dodiya, Reham Fatima, Astrid Gall, Carlos Garcia Giron, Laurent Gil, Tiago Grego, Leanne Haggerty, Erin Haskell, Thibaut Hourlier, Osagie G. Izuogu, Sophie H. Janacek, Thomas Juettemann, Mike Kay, Ilias Lavidas, Tuan Le, Diana Lemos, Jose Gonzalez Martinez, Thomas Maurel, Mark McDowall, Aoife McMahon, Shamika Mohanan, Benjamin Moore, Michael Nuhn, Denye N. Oheh, Anne Parker, Andrew Parton, Mateus Patricio, Manoj Pandian Sakthivel, Ahamed Imran Abdul Salam, Bianca M. Schmitt, Helen Schuilenburg, Dan Sheppard, Mira Sycheva, Marek Szuba, Kieron Taylor, Anja Thormann, Glen Threadgold, Alessandro Vullo, Brandon Walts, Andrea Winterbottom, Amonida Zadissa, Marc Chakiachvili, Bethany Flint, Adam Frankish, Sarah E. Hunt, Garth IIsley, Myrto Kostadima, Nick Langridge, Jane E. Loveland, Fergal J. Martin, Joannella Morales, Jonathan M. Mudge, Matthieu Muffato, Emily Perry, 
Magali Ruffier, Stephen J. Trevanion, Fiona Cunningham, Kevin L. Howe, Daniel R. Zerbino, and Paul Flicek. Ensembl 2020. Nucleic Acids Research, 48(D1):D682-D688, January 2020. ISSN 1362-4962. doi: 10.1093/nar/gkz966.

Jiaxin Zhu, Tiantian Zhang, Jiahao Li, Junming Lin, Wenhua Liang, Wenjie Huang, Ning Wan, and Jie Jiang. Association Between Tumor Mutation Burden (TMB) and Outcomes of Cancer Patients Treated With PD-1/PD-L1 Inhibitions: A Meta-Analysis. Frontiers in Pharmacology, 10:673, 2019. ISSN 1663-9812. doi: 10.3389/fphar.2019.00673. 